\title{
Electrochemical behaviour and structure of novel phosphine- and phosphite-substituted tungsten(0) Fischer carbene complexes
}

\author{
Marilé Landman ${ }^{a} *$, René Pretorius, ${ }^{a}$ Roan Fraser, ${ }^{a}$ Blenerhassit E. Buitendach, ${ }^{b}$ \\ Marrigje M. Conradie, ${ }^{b}$ Petrus H. van Rooyen ${ }^{a}$ and Jeanet Conradie $e^{b}$
}

${ }^{a}$ Department of Chemistry, University of Pretoria, Private Bag X20, Hatfield, 0028, South Africa. Tel: 27-12-4202527 Fax: 27-12-4204687

b Department of Chemistry, PO Box 339, University of the Free State, Bloemfontein, 9300, South Africa. Tel: 27-51-4012194, Fax: 27-51-4446384

Contact author details:

Name: Marilé Landman Tel: ++27-12-4202527 Fax: ++27-12-4204687, email: marile.landman@up.ac.za

Name: Jeanet Conradie, Tel: ++27-51-4012194, Fax: ++27-51-4446384, email: conradj@ufs.ac.za

Dedicated to the memory of Professor Robert Vleggaar (1945-2013), our friend and colleague

\section{Research highlights}

- Synthesis and crystal structure of novel phosphine-substituted Fischer ethoxy-Wcarbenes

- DFT understanding of oxidation and reduction centre of W-carbenes

- Relationship between redox potentials and DFT calculated energies of W-carbenes

\section{Abstract}

The electrochemical behaviour of a series of sixteen novel Fischer ethoxy- and aminocarbene complexes of the type $\left[(\mathrm{CO})_{4}\left(\mathrm{PPh}_{3}\right) \mathrm{W}=\mathrm{C}(\mathrm{X}) \mathrm{R}\right]$ with $\mathrm{R}=2$-thienyl or 2furyl and $\mathrm{X}=\mathrm{OEt}$ or NHCy, (1-4), $\left[(\mathrm{CO})_{3}(\mathrm{dppe}) \mathrm{W}=\mathrm{C}(\mathrm{X}) \mathrm{R}\right]$ with $\mathrm{R}=2$-thienyl or 2furyl and $\mathrm{X}=\mathrm{OEt}$ or NHCy, (5-8) (dppe = 1,2-bis(diphenylphosphino)ethane) and 
$\left[(\mathrm{CO})_{4} \mathrm{P}(\mathrm{OPh})_{3} \mathrm{~W}=\mathrm{C}(\mathrm{X}) \mathrm{R}\right]$ with $\mathrm{R}=2$-thienyl or 2-furyl and $\mathrm{X}=\mathrm{OEt}$ or NHCy, (9-12) is investigated by means of cyclic voltammetry. The complexes all exhibit a twoelectron oxidation process that is $\mathrm{W}$-based and a one-electron reduction that is mainly localized on the carbene ligand. Complexes 1-4 and 9-12 are considerably more difficult to oxidize than 5-8 due to the better $\pi$-acceptor ability of the $(\mathrm{CO})_{4}\left(\mathrm{PR}_{3}\right)\left(\mathrm{R}^{\prime}\right.$ $=\mathrm{Ph}$ or $\mathrm{OPh})$ ligand combination than that of $(\mathrm{CO})_{3}(\mathrm{dppe})$. Density functional theory calculations on the neutral, reduced and oxidized complexes confirmed the role of the frontier orbitals in the oxidation and reduction processes and enabled formulation of mathematical relationships that can be used to predict experimental measured potentials. X-ray crystal structures of $\mathbf{2 c i s ,} \mathbf{3}$ and $\mathbf{5}$ are discussed.

\section{Keywords}

Fischer carbene, Tungsten, Phosphine, Electrochemistry, DFT

\section{Introduction}

Recently, electrochemical studies were reported on the behaviour of $\operatorname{Cr}(0)$ [1-3] and W(0) [4] pentacarbonyl Fischer carbene complexes. In these studies, both carbene ligand substituents, i.e. the heteroarene substituent as well as the heteroatom substituent were varied. In this study, this work was extended to determine the effect of modification of the metal ligand environment on the electrochemical behaviour of tungsten Fischer carbene complexes. Towards this end, a series of sixteen novel tungsten(0) Fischer carbene complexes are synthesized in which one or two carbonyl groups are substituted with phosphines or phosphites.

Modification of the ligand sphere of carbonyl carbene complexes was studied not long after the discovery of the first metal carbene complex [5-7]. Substitution of CO ligands while leaving the carbene ligand intact is achieved with phosphines. It was noted by Fischer that phosphine ligands allowed the complexes higher stability in air than their carbonyl analogues [7]. Two possible reaction pathways are observed for the reaction of phosphines with metal pentacarbonyl carbenes. In the first case, rate limiting dissociation of a carbonyl ligand precedes rapid coordination of the phosphine to the metal in a ligand substitution reaction. In the second case, nucleophilic attack of the phosphine at the carbene carbon leads to ylide formation 
and subsequent substitution of the carbene ligand by a phosphine to yield $\mathrm{M}(\mathrm{CO})_{5} \mathrm{PR}_{3}$. Phosphines of low nucleophilicity, e.g. $\mathrm{PPh}_{3}$, tend to follow the first pathway while smaller, more basic phosphines, e.g. $\mathrm{PMe}_{3}$, follow the second route.

The $\sigma$-donor, $\pi$-acceptor [8] and steric [9] characteristics of phosphines can be regulated by modification of their substituents. Phosphines have many applications in organometallic chemistry, specifically as ligands in homogenous catalysts [10]. In the case of Fischer carbenes, phosphine "chelates", which are tethered to the carbene through the substituent on the carbene ligand [11], have been synthesised, as well as examples of carbenes bound to polymers through the phosphorous ligand [12,13]. It was shown that phosphines confer additional reactivity to the carbene complex during reactions with electrophiles, and alter the reactivity pattern during certain organic transformations by varying the stereoselectivity of the complex [14]. The change in stereoselectivity was brought about by the use of a chiral phosphine. Barluenga et. al. [15] also noted that phosphite ligands can be used to introduce chirality into a Fischer carbene complex. Phosphine carbene complexes were used by Arrieta et.al. [16] to alter the reactivity of Fischer carbene complexes during photocarbonylation reactions and to better understand the reaction mechanism. Even though these modifications of Fischer carbene complexes have been known since the late 1960s, not many examples of these complexes were found in literature. This gap was especially noticeable for the diphosphine derivatives [17]. Furthermore, to our knowledge, no examples of heteroaryl phosphine carbenes are known. The combination of altered reactivity of the complex and the catalytic properties of phosphines, motivated this investigation.

In order to design a Fischer carbenes of certain reactivity, the influence of different substituents, heteroatoms and groups on the electrochemical properties of the whole molecule has to be quantified. Cyclic voltammetry (CV) is one of the most versatile electroanalytical techniques to study electroactive species [18-20]. The oxidation and reduction potentials of a species can be measured with relative ease over a wide potential range. Electrochemistry thus represents an important experimental tool to the description and understanding of the molecular electronic structure and redox properties of a complex. The electronic influence of the different substituents, heteroatoms and groups on the redox properties of the Fischer carbene can be expressed qualitatively by correlation with electrochemical data. Theoretical density 
functional theory (DFT) calculation of the energies of the frontier MOs involved in the oxidation and reduction of the complex can be used as a tool to quantitatively correlate experimental and calculated data of a series of related complexes [21-23]. To this extend we hereby present a combined experimental, structural, electrochemical and DFT study of a series of phosphine tungsten carbene complexes.

\section{Experimental}

\subsection{General}

All reactions, unless otherwise noted, were performed under inert nitrogen or argon atmospheres using standard Schlenk techniques [24]. All solvents were freshly distilled, dried and collected under inert conditions, with the exception of toluene. Toluene was not dried, but used after bubbling nitrogen gas through the solvent for 5 to 10 minutes. Column chromatography was carried out under inert nitrogen and argon atmospheres using silica gel (particle size 0.063-0.200 $\mathrm{mm}$ ) as the stationary phase. Percentage yields were calculated relative to the limiting reactant. Crystallization was done using hexane:DCM diffusion methods. Commercial thiophene was purified [25] and triethyloxonium tetrafluoroborate [26] was prepared according to a reported literature procedure. The reagents $\mathrm{W}(\mathrm{CO})_{6}$, n-butyl lithium (1.6 M solution in hexane), furan, 1,2-bis(diphenylphosphino)ethane (dppe), $\mathrm{PPh}_{3}$, cyclohexyl amine (CHA) and other commercial reagents were used as purchased. NMR spectra were recorded on a Bruker ARX-300. NMR spectra were recorded in $\mathrm{CDCl}_{3}, \mathrm{CD}_{3} \mathrm{CN}$ or $\mathrm{CD}_{2} \mathrm{Cl}_{2}$ using deuterated solvent peaks as the internal references. ${ }^{1} \mathrm{H},{ }^{13} \mathrm{C}$ and ${ }^{31} \mathrm{P}$ NMR spectra were measured at 300.1, 75.5 and $81 \mathrm{MHz}$, respectively. IR spectra were recorded on a Perkin Elmer Spectrum RXI FT-IR spectrophotometer as $\mathrm{KBr}$ pellets and only the vibration bands in the carbonyl stretching region ( $c a$. $1500-2200 \mathrm{~cm}^{-1}$ ) are reported. Mass spectra were recorded on a SYNAPT G2 HDMS with the TOF-MS method with sampling time of 4 minutes, with direct infusion inlet method. The source was electron spray ionization. 
The unsubstituted pentacarbonyl carbene precursor complexes A [27], B [28], C [4] and D [4] were prepared according to literature procedures and spectroscopic characterization data follow:

A: Yield: 82.6\%, bright orange solid. - ${ }^{1} \mathrm{H}$ NMR $\left(\mathrm{CDCl}_{3}, \mathrm{ppm}\right): \delta 7.80$ (dd, H10, 5.0, $0.6 \mathrm{~Hz}), 8.14$ (dd, H8, 4.1, $0.8 \mathrm{~Hz}$ ), 7.20 (dd, H9, 4.8, $4.3 \mathrm{~Hz}), 4.98$ (q, H11, 7.1 Hz), 1.64 (t, H12, $7.1 \mathrm{~Hz}) .-{ }^{13} \mathrm{C} \mathrm{NMR}\left(\mathrm{CDCl}_{3}, \mathrm{ppm}\right): \delta 290.7$ (C6), 202.5 (s, CO trans), $197.6\left(\mathrm{t}, \mathrm{CO}_{\text {cis }}, \mathrm{J}_{\mathrm{W}-\mathrm{C}}=126.4 \mathrm{~Hz}\right), 158.3(\mathrm{C} 7), 136.5$ (C10), 141.5 (C8), 129.0 (C9), $78.6(\mathrm{C} 11), 15.0(\mathrm{C} 12)$. - IR $\left(\mathrm{KBr}, \mathrm{cm}^{-1}\right): v_{\mathrm{CO}}=2066(\mathrm{~m}), 1916(\mathrm{vs})$.

B: Yield: $73.3 \%$, bright orange solid. - ${ }^{1} \mathrm{H} \mathrm{NMR}\left(\mathrm{CDCl}_{3}, \mathrm{ppm}\right): \delta 7.86$ (dd, H10, 1.6, $0.7 \mathrm{~Hz}), 7.13$ (dd, H8, 3.7, $0.7 \mathrm{~Hz}), 6.60$ (dd, H9, 3.7, $1.7 \mathrm{~Hz}), 4.94$ (q, H11, 7.1 Hz), 1.62 (t, H12, 7.1 Hz).- ${ }^{13} \mathrm{C}$ NMR ( $\left.\mathrm{CDCl}_{3}, \mathrm{ppm}\right): \delta 284.8$ (C6), $203.4\left(\mathrm{CO}_{\text {trans }}\right), 197.4$ ( $\mathrm{CO}_{\text {cis }}$ ), 166.2 (C7), 150.0 (C10), 113.4 (C8), 113.4 (C9), 78.4 (C11), 15.1 (C12).- IR $\left(\mathrm{KBr}, \mathrm{cm}^{-1}\right): v_{\mathrm{CO}}=2067(\mathrm{~m}), 1920(\mathrm{vs})$.

C: Yield: $88.2 \%$, bright orange yellow solid MS $(\mathrm{m} / z): 433$ ([M] $\left.{ }^{+}-3 \mathrm{CO}\right) ; 280\left([\mathrm{M}]^{+}-5 \mathrm{CO}-\mathrm{CHA}\right)$. - ${ }^{1} \mathrm{H}$ NMR $\left(\mathrm{CDCl}_{3}, \mathrm{ppm}\right)$ : Isomer I: $\delta 8.30(\mathrm{~s}(\mathrm{~b}), \mathrm{N}-\mathbf{H}), 7.50(\mathrm{dd}, \mathrm{H} 10,5.1,1.2 \mathrm{~Hz}), 7.34(\mathrm{dd}, \mathrm{H} 8,3.8,1.2 \mathrm{~Hz}), 7.12(\mathrm{dd}$, H9, 5.1, 3.8 Hz), 4.35-4.52 (m, H11), 1.10-2.20 (m, Cyclohexyl-NH); Isomer II: $\delta$ 8.64 (s(b), N-H), 7.46 (dd, H10, 5.0, 1.1 Hz), 7.07 (dd, H9, 5.0, 3.7 Hz), 6.93 (dd, H8, 3.7, 1.1 Hz), 3.73-3.86 (m, H11), 1.10-2.20 (m, Cyclohexyl-NH).- ${ }^{13} \mathrm{C}$ NMR $\left(\mathrm{CDCl}_{3}\right.$, ppm): Isomer I: $\delta 235.0\left(\mathrm{t}, \mathrm{C} 6, \mathrm{~J}_{\mathrm{W}-\mathrm{C}}=90.8 \mathrm{~Hz}\right), 202.8\left(\mathrm{t}, \mathrm{CO}_{\text {trans }}, \mathrm{J}_{\mathrm{W}-\mathrm{C}}=127.1 \mathrm{~Hz}\right)$, $198.2\left(\mathrm{t}, \mathrm{CO}_{\text {cis }}, \mathrm{J}_{\mathrm{W}-\mathrm{C}}=127.0 \mathrm{~Hz}\right), 157.0$ (C7), 129.5 (C10), 128.1 (C8), 126.9 (C9), 64.9 (C11), 33.0 (C12), 24.9 (C13), 24.4 (C14). Isomer II: $\delta 245.7$ (t, C6, J J-C $=90.8$ $\mathrm{Hz}), 203.5\left(\mathrm{t}, \mathrm{CO}_{\text {trans }}, \mathrm{J}_{\mathrm{W}-\mathrm{C}}=127.8 \mathrm{~Hz}\right), 198.7\left(\mathrm{t}, \mathrm{CO}_{\text {cis }}, \mathrm{J}_{\mathrm{W}-\mathrm{C}}=127.8 \mathrm{~Hz}\right), 150.2(\mathrm{C} 7)$, 127.8 (C10), 127.3 (C8), 124.2 (C9), 59.5 (C11), 33.3 (C12), 24.7 (C13), 24.2 (C14).IR $\left(\mathrm{KBr}, \mathrm{cm}^{-1}\right): v_{\mathrm{CO}}=2061(\mathrm{~m}), 1972(\mathrm{vw}), 1950(\mathrm{~s}), 1906(\mathrm{vs})$.

D: Yield: $94.6 \%$, bright yellow solid MS $(m / z): 280\left([\mathrm{M}]^{+}-5 \mathrm{CO}-C y c l o h e x y l\right) .-{ }^{1} \mathrm{H} \mathrm{NMR}\left(\mathrm{CDCl}_{3}, \mathrm{ppm}\right)$ : Isomer I: $\delta 8.93$ (s(b), N-H), 7.51 (d, H10, 1.4 Hz), 7.38 (dd, H8, 3.6, 0.5 Hz), 6.57 (dd, H9, 3.6, 0.5 Hz), 4.31-4.42 (m, H11), 1.12-2.15 (m, Cyclohexyl-NH); Isomer II: $\delta 8.13$ (s(b), NH), 7.68 (dd, H10, 1.8, 0.6 Hz), 7.25 (dd, H8, 3.6, 0.6 Hz), 6.60 (dd, H9, 3.6, $1.8 \mathrm{~Hz})$,

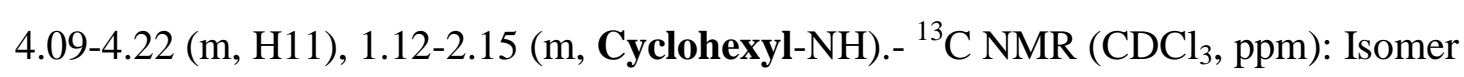
I: $\delta 219.3(\mathrm{C} 6), 202.4\left(\mathrm{CO}_{\text {trans }}\right), 198.4\left(\mathrm{t}, \mathrm{CO}_{\mathrm{cis}}, \mathrm{J}_{\mathrm{W}-\mathrm{C}}=126.9 \mathrm{~Hz}\right), 159.1(\mathrm{C} 7), 144.1$ 
(C10), 126.3 (C8), 113.7 (C9), 64.3 (C11), 33.1 (C12), 24.5 (C13), 25.3 (C14). Isomer II: $\delta 225.7$ (C6), $202.8\left(\mathrm{CO}_{\text {trans }}\right), 199.1\left(\mathrm{t}, \mathrm{CO}_{\text {cis }}, \mathrm{J}_{\mathrm{W}-\mathrm{C}}=122.2 \mathrm{~Hz}\right), 157.6(\mathrm{C} 7), 145.8$ (C10), 126.4 (C8), 113.3 (C9), 61.1 (C11), 33.2 (C12), 24.3 (C13), 25.3 (C14).- IR $\left(\mathrm{KBr}, \mathrm{cm}^{-1}\right): v_{\mathrm{CO}}=2059(\mathrm{~m}), 1966(\mathrm{vw}), 1908(\mathrm{~s}), 1882(\mathrm{vs})$.

\subsubsection{Synthesis of cis- and trans-[W(PPh $\left.)(\mathrm{CO})_{4}\left\{\mathrm{C}(\mathrm{OEt}) \mathrm{C}_{4} \mathrm{H}_{4} \mathrm{~S}\right\}\right]$, (1cis) and (1trans)}

Complex A (1.393 g, $3.00 \mathrm{mmol})$ and $\mathrm{PPh}_{3}(0.945 \mathrm{~g}, 3.60 \mathrm{mmol})$ were dissolved in 30 $\mathrm{mL}$ of toluene (wine-red solution). This mixture was left to reflux overnight (dark brown solution), and the solvent was removed (dark brown solid). The crude product was purified on silica gel using hexane:DCM gradients. Unreacted carbene A $(0.285$ g, $0.614 \mathrm{mmol}, 20.5 \%$ ) and two products were obtained according to TLC. The two products were identified as the cis and trans isomers of the product. The trans product, 1trans, is yellow-brown in solution and elutes after the starting carbene $\mathbf{A}$. The cis product, 1cis, eluted directly after the trans isomer as a dark red-brown solution. The bands corresponding to the isomers could not be completely separated and thus fractions were collected and combined as necessary.

1cis: Yield: $0.590 \mathrm{~g}, 0.845 \mathrm{mmol}, 28.2 \%$, reddish-brown solid $\operatorname{MS}(\mathrm{m} / z): 557$ ([M] $\left.{ }^{+}-\mathrm{Et}-4 \mathrm{CO}\right) ; 327\left([\mathrm{M}]^{+}-4 \mathrm{Ph}-\mathrm{Et}-4 \mathrm{CO}\right) .-{ }^{1} \mathrm{H} \mathrm{NMR}\left(\mathrm{CD}_{2} \mathrm{Cl}_{2}, \mathrm{ppm}\right): \delta$ 7.77 (dd, H8, 4.0, $1.1 \mathrm{~Hz}$ ), 7.65 (dd, H10, 5.0, $1.1 \mathrm{~Hz}$ ), 6.97 (dd, H9, 4.8, 4.0 Hz), 7.31-7.46 (m, PPh $), 4.53$ (dq, H11, 7.0 Hz, J $\left.\mathrm{P}_{\mathrm{H}}=0.5 \mathrm{~Hz}\right), 1.20(\mathrm{t}, \mathrm{H} 12,7.0 \mathrm{~Hz}) .{ }^{13} \mathrm{C}$ NMR ( $\left.\mathrm{CD}_{2} \mathrm{Cl}_{2}, \mathrm{ppm}\right): \delta 294.5\left(\mathrm{~d}, \mathrm{C} 6, \mathrm{~J}_{\mathrm{P}-\mathrm{C}}=7.0 \mathrm{~Hz}\right), 211.4\left(\mathrm{~d}, \mathrm{CO} 1, \mathrm{~J}_{\mathrm{P}-\mathrm{C}}=6.5 \mathrm{~Hz}\right)$, $207.3\left(\mathrm{~d}, \mathrm{CO} 3, \mathrm{~J}_{\mathrm{P}-\mathrm{C}}=24.0 \mathrm{~Hz}\right), 203.8\left(\mathrm{~d}, \mathrm{CO} 2, \mathrm{~J}_{\mathrm{P}-\mathrm{C}}=6.8 \mathrm{~Hz}\right), 159.9(\mathrm{C} 7), 140.2(\mathrm{C} 8)$, 133.1 (C10), 128.7 (C9), 135.9 (d, PhC, $\left.\mathrm{J}_{\mathrm{P}-\mathrm{C}}=38.3 \mathrm{~Hz}\right), 133.7\left(\mathrm{~d}, \mathrm{PhC}_{\text {ortho }}, \mathrm{J}_{\mathrm{P}-\mathrm{C}}=11.8\right.$ $\mathrm{Hz}), 130.4\left(\mathrm{~d}, \mathrm{PhC}_{\text {para }}, \mathrm{J}_{\mathrm{P}-\mathrm{C}}=1.7 \mathrm{~Hz}\right), 129.0\left(\mathrm{~d}, \mathrm{PhC}_{\text {meta }}, \mathrm{J}_{\mathrm{P}-\mathrm{C}}=9.7 \mathrm{~Hz}\right), 77.8(\mathrm{~d}, \mathrm{C} 11$, $\left.\mathrm{J}_{\mathrm{P}-\mathrm{C}}=1.8 \mathrm{~Hz}\right), 14.8(\mathrm{C} 12) .{ }^{31} \mathrm{P}$ NMR $\left(\mathrm{CD}_{2} \mathrm{Cl}_{2}, \mathrm{ppm}\right): \delta 25.00\left(\mathrm{~d}, \mathbf{P P h}_{3}, \mathrm{~J}_{\mathrm{P}-\mathrm{W}}=233.6\right.$ Hz). - IR (KBr, cm $\left.{ }^{-1}\right): v_{\mathrm{CO}}=2009$ (s), 1914 (s), 1894 (s), 1883 (s).

1trans: Yield: $0.040 \mathrm{~g}, 0.057 \mathrm{mmol}, 1.9 \%$, yellow-brown solid $\operatorname{MS}(\mathrm{m} / \mathrm{z}): 352\left([\mathrm{M}]^{+}-\mathrm{Et}-\mathrm{PPh}_{3}-2 \mathrm{CO}\right) ; 327\left([\mathrm{M}]^{+}-4 \mathrm{Ph}-\mathrm{Et}-4 \mathrm{CO}\right) .-{ }^{1} \mathrm{H}$ NMR $\left(\mathrm{CD}_{2} \mathrm{Cl}_{2}\right.$, ppm): $\delta 8.01\left(\mathrm{dd}, \mathrm{H} 8,4.0,1.1 \mathrm{~Hz}, \mathrm{~J}_{\mathrm{P}-\mathrm{H}}=0.3 \mathrm{~Hz}\right), 7.65\left(\mathrm{ddd}, \mathrm{H} 10,5.1,1.1 \mathrm{~Hz}, \mathrm{~J}_{\mathrm{P}-\mathrm{H}}=\right.$ $0.6 \mathrm{~Hz}), 7.15$ (dd, H9, 5.1, 4.0 Hz), 7.32-7.49 (m, PPh 3 , meta and para), 7.50-7.58 (m, $\mathrm{PPh}_{3}$, ortho), 4.98 (q, H11, 7.0 Hz), 1.61 (t, H12, 7.0 Hz). ${ }^{13} \mathrm{C} \mathrm{NMR}\left(\mathrm{CD}_{2} \mathrm{Cl}_{2}, \mathrm{ppm}\right)$ : $\delta 283.7\left(\mathrm{~d}, \mathrm{C} 6, \mathrm{~J}_{\mathrm{P}-\mathrm{C}}=15.3 \mathrm{~Hz}\right), 205.2\left(\mathrm{~d}, \mathrm{CO}, \mathrm{J}_{\mathrm{P}-\mathrm{C}}=6.4 \mathrm{~Hz}\right), 161.1(\mathrm{C} 7), 138.9(\mathrm{C} 8)$, 
131.9 (C10), 128.9 (C9), 137.8 (d, PhC, $\left.\mathrm{J}_{\mathrm{P}-\mathrm{C}}=38.9 \mathrm{~Hz}\right), 133.7\left(\mathrm{~d}, \mathrm{PhC}_{\text {ortho }}, \mathrm{J}_{\mathrm{P}-\mathrm{C}}=11.8\right.$ $\mathrm{Hz}), 130.2\left(\mathrm{~d}, \mathrm{PhC}_{\text {para }}, \mathrm{J}_{\mathrm{P}-\mathrm{C}}=1.6 \mathrm{~Hz}\right), 128.9\left(\mathrm{~d}, \mathrm{PhC}_{\text {meta }}, \mathrm{J}_{\mathrm{P}-\mathrm{C}}=9.5 \mathrm{~Hz}\right), 76.2(\mathrm{~d}, \mathrm{C} 11)$,

15.4 (C12). - ${ }^{31} \mathrm{P}$ NMR $\left(\mathrm{CD}_{2} \mathrm{Cl}_{2}, \mathrm{ppm}\right): \delta 22.78\left(\mathrm{~d}, \mathbf{P P h}_{3}, \mathrm{~J}_{\mathrm{P}-\mathrm{W}}=209.3 \mathrm{~Hz}\right)$. - IR $(\mathrm{KBr}$, $\left.\mathrm{cm}^{-1}\right): v_{\mathrm{CO}}=2017(\mathrm{vw}), 1944(\mathrm{w}), 1892$ (s). Anal. Calcd for $\mathrm{C}_{29} \mathrm{H}_{23} \mathrm{O}_{5} \mathrm{PSW}: \mathrm{C}, 49.87$; H, 3.32. Found: C, 51.02; H, 3.13.

\subsubsection{Synthesis of cis- and trans $-\left[\mathrm{W}\left(\mathrm{PPh}_{3}\right)(\mathrm{CO})_{4}\left\{\mathrm{C}(\mathrm{OEt}) \mathrm{C}_{4} \mathrm{H}_{4} \mathrm{O}\right\}\right]$, (2cis) and (2trans)}

Complex B (0.868 g, $1.94 \mathrm{mmol})$ and $\mathrm{PPh}_{3}(0.566 \mathrm{~g}, 2.14 \mathrm{mmol})$ were dissolved in 40 $\mathrm{mL}$ of toluene (wine red solution) and left to reflux for 7 hours (brown solution). The solvent was then removed, yielding a brown solid as the crude product. TLC indicated starting monocarbene $\mathbf{B}$ and two products, cis and trans isomers, were present. Starting monocarbene $\mathbf{B}$ was removed from the products on a silica gel filter using hexane:DCM gradient elution. The isomers were then separated on a cooled silica gel column using hexane:DCM gradient elution. Elution patterns and colours for the isomers were the same as for the thiophene analogues.

2cis: Yield: 0.615 g, $0.901 \mathrm{mmol}, 46.6 \%$, red-brown solid MS $(m / z): 654\left[\mathrm{M}^{+} ; 541\left([\mathrm{M}]^{+}-4 \mathrm{CO}-\mathrm{Et}\right), 310\left([\mathrm{M}]^{+}-3 \mathrm{Ph}-4 \mathrm{CO}-\mathrm{Et}\right), 308\left([\mathrm{M}]^{+}-\mathrm{PPh}_{3^{-}}\right.\right.$ 4CO), 279 ([M] $\left.{ }^{+}-\mathrm{PPh}_{3}-4 \mathrm{CO}-\mathrm{Et}\right) .-{ }^{1} \mathrm{H}$ NMR $\left(\mathrm{CD}_{2} \mathrm{Cl}_{2}, \mathrm{ppm}\right): \delta$ 7.30-7.43 (m, H10),

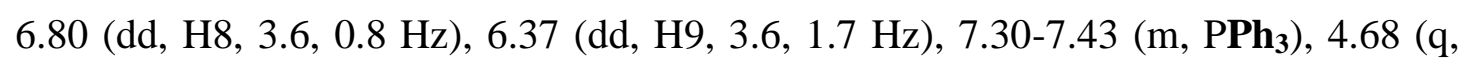
$\mathrm{H} 11,7.1 \mathrm{~Hz}), 1.36$ (t, H12, $7.1 \mathrm{~Hz}) .{ }^{13} \mathrm{C} \mathrm{NMR}\left(\mathrm{CD}_{2} \mathrm{Cl}_{2}, \mathrm{ppm}\right): \delta 289.0\left(\mathrm{dd}, \mathrm{C} 6, \mathrm{~J}_{\mathrm{P}-\mathrm{C}}\right.$ $\left.=6.9 \mathrm{~Hz}, \mathrm{~J}_{\mathrm{W}-\mathrm{C}}=105.4 \mathrm{~Hz}\right), 212.3\left(\mathrm{dt}, \mathrm{CO} 1, \mathrm{~J}_{\mathrm{P}-\mathrm{C}}=6.7 \mathrm{~Hz}, \mathrm{~J}_{\mathrm{W}-\mathrm{C}}=115.5 \mathrm{~Hz}\right), 207.3(\mathrm{dt}$, $\left.\mathrm{CO} 3, \mathrm{~J}_{\mathrm{P}-\mathrm{C}}=24.4 \mathrm{~Hz}, \mathrm{~J}_{\mathrm{W}-\mathrm{C}}=146.7 \mathrm{~Hz}\right), 203.8\left(\mathrm{dt}, \mathrm{CO} 2, \mathrm{~J}_{\mathrm{P}-\mathrm{C}}=6.9 \mathrm{~Hz}, \mathrm{~J}_{\mathrm{W}-\mathrm{C}}=128.0\right.$ $\mathrm{Hz}$ ), 167.1 (C7), 148.5 (C10), 113.1 (C8), 112.1 (C9), 136.5 (d, PhC, J $\mathrm{J}_{\mathrm{P}-\mathrm{C}}=38.2 \mathrm{~Hz}$ ), $133.8\left(\mathrm{~d}, \mathrm{PhC}_{\text {ortho }}, \mathrm{J}_{\mathrm{P}-\mathrm{C}}=11.8 \mathrm{~Hz}\right), 130.4\left(\mathrm{~d}, \mathrm{PhC}_{\text {para }}, \mathrm{J}_{\mathrm{P}-\mathrm{C}}=1.7 \mathrm{~Hz}\right), 128.8\left(\mathrm{~d}, \mathrm{PhC}_{\text {meta }}\right.$, $\left.\mathrm{J}_{\mathrm{P}-\mathrm{C}}=9.5 \mathrm{~Hz}\right), 77.4(\mathrm{C} 11), 15.3(\mathrm{C} 12) .-{ }^{31} \mathrm{P} \mathrm{NMR}\left(\mathrm{CD}_{2} \mathrm{Cl}_{2}, \mathrm{ppm}\right): \delta 25.25\left(\mathrm{~d}, \mathbf{P P h}_{3}\right.$, $\left.\mathrm{J}_{\mathrm{P}-\mathrm{W}}=236.3 \mathrm{~Hz}\right)$. - IR (KBr, cm $\left.{ }^{-1}\right): v_{\mathrm{CO}}=2009(\mathrm{~s}), 1921$ (s), 1888 (s), 1860 (s).

2trans: Yield: $0.030 \mathrm{~g}, 0.044 \mathrm{mmol}, 3.3 \%$, yellow-brown solid

${ }^{1} \mathrm{H}$ NMR $\left(\mathrm{CD}_{2} \mathrm{Cl}_{2}, \mathrm{ppm}\right): \delta 7.79$ (s, H10), 6.97 (d, H8, $3.5 \mathrm{~Hz}$ ), 6.55 (dd, H9, 3.5, 1.5 $\mathrm{Hz}$ ), 7.32-7.48 (m, $\mathbf{P P h}_{3}$, meta and para), 7.52-7.60 (m, PPh 3 ,ortho), 4.97 (q, H11, 7.0 $\mathrm{Hz}), 1.60$ (t, H12, 7.0 Hz). - ${ }^{13} \mathrm{C} \mathrm{NMR}\left(\mathrm{CD}_{2} \mathrm{Cl}_{2}, \mathrm{ppm}\right): \delta 278.5(\mathrm{C} 6), 205.0\left(\mathrm{~d}, \mathrm{CO}, \mathrm{J}_{\mathrm{P}-}\right.$ $\mathrm{C}=5.5 \mathrm{~Hz}), 167.5(\mathrm{C} 7), 147.8(\mathrm{C} 10), 113.1(\mathrm{C} 8), 111.2(\mathrm{C} 9), 138.1\left(\mathrm{~d}, \mathrm{PhC}, \mathrm{J}_{\mathrm{P}-\mathrm{C}}=\right.$ $37.6 \mathrm{~Hz}), 134.0\left(\mathrm{~d}, \mathrm{PhC}_{\text {ortho }}, \mathrm{J}_{\mathrm{P}-\mathrm{C}}=11.3 \mathrm{~Hz}\right), 130.3\left(\mathrm{PhC}_{\text {para }}\right), 129.0\left(\mathrm{~d}, \mathrm{PhC}_{\text {meta }}, \mathrm{J}_{\mathrm{P}-\mathrm{C}}=\right.$ 
9.1 Hz), 76.0 (d, C11), 15.6 (C12). - ${ }^{31} \mathrm{P}$ NMR $\left(\mathrm{CD}_{2} \mathrm{Cl}_{2}, \mathrm{ppm}\right): \delta 23.00\left(\mathrm{~d}, \mathbf{P P h}_{3}, \mathrm{~J}_{\mathrm{P}-\mathrm{W}}\right.$ $=209.3 \mathrm{~Hz})$. - IR $\left(\mathrm{KBr}, \mathrm{cm}^{-1}\right): v_{\mathrm{CO}}=2019(\mathrm{vw}), 1941(\mathrm{w}), 1884(\mathrm{~s})$. Anal. Calcd for $\mathrm{C}_{29} \mathrm{H}_{23} \mathrm{O}_{6} \mathrm{PW}$ : C, 51.05; H, 3.40. Found: C, 51.02; H, 3.23.

\subsubsection{Synthesis of cis $-\left[\mathrm{W}\left(\mathrm{PPh}_{3}\right)(\mathrm{CO})_{4}\left\{\mathrm{C}(\mathrm{CHA}) \mathrm{C}_{4} \mathrm{H}_{4} \mathrm{~S}\right\}\right]$, (3)}

Complex $\mathbf{C}(0.263 \mathrm{~g}, 0.51 \mathrm{mmol})$ and $\mathrm{PPh}_{3}(0.264 \mathrm{~g}, 1.01 \mathrm{mmol})$ were dissolved in 40 $\mathrm{mL}$ of toluene, resulting in a bright yellow solution. The reaction mixture was allowed to reflux, with stirring for 4 hours. At this point, decomposition products were evident as a brown precipitate was formed, and thus the solution was cooled immediately and the solvent removed. A bright orange solid was obtained. The crude product was then purified on a silica gel column using hexane:DCM gradient elution. Two fractions were collected. The first fraction was $\mathbf{1}(0.207 \mathrm{~g}, 0.41 \mathrm{mmol})$. The second fraction was bright orange and contained the product. The product was obtained as a pale orange crystalline solid.

3: Yield: $0.162 \mathrm{~g}, 0.216 \mathrm{mmol}, 42.8 \%$, pale orange crystalline solid

${ }^{1} \mathrm{H}$ NMR $\left(\mathrm{CD}_{2} \mathrm{Cl}_{2}, \mathrm{ppm}\right): \delta 8.10$ (s (b), N-H), 7.33-7.43 (m, H10), 7.12 (dd, H8, 3.7, $1.2 \mathrm{~Hz}), 6.97$ (dd, H9, 5.0, 3.7 Hz), 7.33-7.43 (m, PPh $)$, 4.02-4.13 (m, H11), 1.061.30, 1.58-1.75 (m, Cyclohexyl-NH). - ${ }^{13} \mathrm{C}$ NMR $\left(\mathrm{CD}_{2} \mathrm{Cl}_{2}, \mathrm{ppm}\right): \delta 245.2$ (d, C6, J $\mathrm{P}-\mathrm{C}$ $=6.9 \mathrm{~Hz}), 210.6\left(\mathrm{~d}, \mathrm{CO} 1, \mathrm{~J}_{\mathrm{P}-\mathrm{C}}=5.4 \mathrm{~Hz}\right), 208.2\left(\mathrm{~d}, \mathrm{CO} 3, \mathrm{~J}_{\mathrm{P}-\mathrm{C}}=25.6 \mathrm{~Hz}\right), 204.6(\mathrm{~d}$, $\left.\mathrm{CO} 2, \mathrm{~J}_{\mathrm{P}-\mathrm{C}}=6.8 \mathrm{~Hz}\right), 159.0(\mathrm{C} 7), 128.2(\mathrm{C} 10), 127.6(\mathrm{C} 8), 127.3(\mathrm{C} 9), 137.0(\mathrm{~d}, \mathrm{PhC}$, $\left.\mathrm{J}_{\mathrm{P}-\mathrm{C}}=36.4 \mathrm{~Hz}\right), 134.1\left(\mathrm{~d}, \mathrm{PhC}_{\text {ortho }}, \mathrm{J}_{\mathrm{P}-\mathrm{C}}=11.8 \mathrm{~Hz}\right), 130.6\left(\mathrm{~s}, \mathrm{PhC}_{\text {para }}\right), 128.9(\mathrm{~d}$, $\left.\mathrm{PhC}_{\text {meta }}, \mathrm{J}_{\mathrm{P}-\mathrm{C}}=9.3 \mathrm{~Hz}\right), 64.0,33.5,25.1,25.7$ (Cyclohexyl-NH). - ${ }^{31} \mathrm{P} \mathrm{NMR}\left(\mathrm{CD}_{2} \mathrm{Cl}_{2}\right.$, ppm): $\delta 24.30\left(\mathrm{~d}, \mathbf{P P h}_{3}, \mathrm{~J}_{\mathrm{P}-\mathrm{W}}=233.0 \mathrm{~Hz}\right) .-\mathrm{IR}\left(\mathrm{KBr}, \mathrm{cm}^{-1}\right): v_{\mathrm{CO}}=2002(\mathrm{~s}), 1894$ (s), 1870 (s), 1860 (s). Anal. Calcd for $\mathrm{C}_{33} \mathrm{H}_{30} \mathrm{NO}_{4} \mathrm{PSW}$ : C, 52.74; H, 4.02; N, 1.86. Found: C, 52.44; H, 4.23; N, 1.99 .

\subsubsection{Synthesis of cis-[W(PPh 3$\left.)(\mathrm{CO})_{4}\left\{\mathrm{C}(\mathrm{CHA}) \mathrm{C}_{4} \mathrm{H}_{4} \mathrm{O}\right\}\right]$, (4)}

Complex $\mathbf{D}(0.256 \mathrm{~g}, 0.51 \mathrm{mmol})$ and $\mathrm{PPh}_{3}(0.264 \mathrm{~g}, 1.01 \mathrm{mmol})$ were dissolved in 40 $\mathrm{mL}$ of toluene, resulting in a bright yellow solution. The reaction mixture was allowed to reflux, with stirring, for 4 hours. A bright orange solid was obtained. The crude product was then purified on a silica gel column using hexane:DCM gradient elution. Two fractions were collected. The first fraction was identified as $\mathbf{2}$, while the second 
fraction was bright orange and contained the product. The product was obtained as a pale orange crystalline solid.

4: Yield: $0.060 \mathrm{~g}, 0.082 \mathrm{mmol}, 39.1 \%$, pale orange crystalline solid

${ }^{1} \mathrm{H}$ NMR $\left(\mathrm{CD}_{2} \mathrm{Cl}_{2}, \mathrm{ppm}\right): \delta 8.75$ (s (b), N-H), 7.21-7.46 (m, H10), 6.82 (d, H8, 3.6), 6.26 (dd, H9, 3.6, 1.7 Hz), 7.21-7.46 (m, PPh $)$, 4.15-4.32 (m, H11), 1.10-1.35, 1.551.80 (m, Cyclohexyl-NH). - ${ }^{13} \mathrm{C}$ NMR $\left(\mathrm{CD}_{2} \mathrm{Cl}_{2}, \mathrm{ppm}\right): \delta 228.8\left(\mathrm{~d}, \mathrm{C} 6, \mathrm{~J}_{\mathrm{P}-\mathrm{C}}=7.8 \mathrm{~Hz}\right)$, $210.1\left(\mathrm{~d}, \mathrm{CO} 1, \mathrm{~J}_{\mathrm{P}-\mathrm{C}}=5.6 \mathrm{~Hz}\right), 208.4\left(\mathrm{~d}, \mathrm{CO} 3, \mathrm{~J}_{\mathrm{P}-\mathrm{C}}=26.5 \mathrm{~Hz}\right), 204.9\left(\mathrm{~d}, \mathrm{CO} 2, \mathrm{~J}_{\mathrm{P}-\mathrm{C}}=\right.$ $6.9 \mathrm{~Hz}), 160.6$ (d, C7, J $\mathrm{P}_{-\mathrm{C}}=1.8 \mathrm{~Hz}$ ), 145.0 (C10), 123.8 (C8), 113.4 (C9), 136.5 (d, $\left.\mathrm{PhC}, \mathrm{J}_{\mathrm{P}-\mathrm{C}}=36.3 \mathrm{~Hz}\right), 134.0\left(\mathrm{~d}, \mathrm{PhC}_{\text {ortho }}, \mathrm{J}_{\mathrm{P}-\mathrm{C}}=11.7 \mathrm{~Hz}\right), 130.3\left(\mathrm{~d}, \mathrm{PhC}_{\text {para }}, \mathrm{J}_{\mathrm{P}-\mathrm{C}}=1.6\right.$ $\mathrm{Hz}), 128.7\left(\mathrm{~d}, \mathrm{PhC}_{\text {meta }}, \mathrm{J}_{\mathrm{P}-\mathrm{C}}=8.9 \mathrm{~Hz}\right), 63.4,33.5,25.0,25.6($ Cyclohexyl-NH$) .-{ }^{31} \mathrm{P}$ NMR $\left(\mathrm{CD}_{2} \mathrm{Cl}_{2}, \mathrm{ppm}\right): \delta 24.10\left(\mathrm{~d}, \mathbf{P P h}_{3}, \mathrm{~J}_{\mathrm{P}-\mathrm{W}}=231.7 \mathrm{~Hz}\right)$. - IR $\left(\mathrm{KBr}, \mathrm{cm}^{-1}\right): v_{\mathrm{CO}}=$ 2001 (s), 1875 (s). Anal. Calcd for $\mathrm{C}_{33} \mathrm{H}_{30} \mathrm{NO}_{5} \mathrm{PW}$ : C, 53.90; H, 4.11; N, 1.90. Found: C, 52.86; H, 4.03; N, 1.81 .

\subsubsection{Synthesis of mer-[W(dppe $\left.)(\mathrm{CO})_{3}\left\{\mathrm{C}(\mathrm{OEt}) \mathrm{C}_{4} \mathrm{H}_{4} \mathrm{~S}\right\}\right], \quad(5)$, and mer- $\left[\mathrm{W}(\mathrm{dppe})(\mathrm{CO})_{3}\left\{\mathrm{C}(\mathrm{OEt}) \mathrm{C}_{4} \mathrm{H}_{4} \mathrm{O}\right\}\right]$, (6)}

Complex A (0.464 g, $1.00 \mathrm{mmol})$ and dppe (0.412 g, $1.03 \mathrm{mmol})$ or Complex B $(0.534 \mathrm{~g}, 1.19 \mathrm{mmol})$ and dppe $(0.748 \mathrm{~g}, 1.88 \mathrm{mmol})$ were dissolved in $30 \mathrm{~mL}$ of toluene (wine red solution). The mixture was then left to reflux until all the starting carbene had reacted (dark brown solution). TLC indicated two products were present; a brown-yellow compound and a more polar brown-red compound. The solvent was removed, yielding a brown solid. The crude product was dissolved in minimal DCM and purified by diffusion crystallization methods with hexane. Large, clumpy, black crystals (mer isomer of target molecule 5 or 6) and fine white crystals of $\mathbf{E}$, [W(CO $\left.)_{4} \mathrm{dppe}\right]$, were obtained. These were separated by sonication of the sample in hexane and then removing the suspension that formed, leaving large black crystals. TLC indicated that the black crystals obtained corresponded to the first yellow-brown compound in the crude product. Separation by silica gel chromatography was attempted, however, the products could not be separated and decomposition occurred; therefore only crystallization was used as a purification method.

5: Yield: 0.312 g, $0.387 \mathrm{mmol}, 38.7 \%$, black crystals MS (m/z): $806\left[\mathrm{M}^{+}\right]$. - ${ }^{1} \mathrm{H}$ NMR $\left(\mathrm{CD}_{2} \mathrm{Cl}_{2}, \mathrm{ppm}\right): \delta$ 7.63-7.70 (m, H10), $7.53(\mathrm{dd}, \mathrm{H} 8$, 3.9, 1.1 Hz), 6.84 (dd, H9, 5.1, 3.9 Hz), 7.30 -7.47, 7.63-7.70 (m, PPh $)$ ), 4.39 (q, H11, 
$7.0 \mathrm{~Hz}), 1.24(\mathrm{t}, \mathrm{H} 12,7.0 \mathrm{~Hz}), 2.53-2.66\left(\mathrm{~m}, \mathrm{C} 14 \mathbf{H}_{2}\right), 2.66-2.82\left(\mathrm{~m}, \mathrm{C} 13 \mathbf{H}_{2}\right) .-{ }^{13} \mathrm{C}$ $\operatorname{NMR}\left(\mathrm{CD}_{2} \mathrm{Cl}_{2}, \mathrm{ppm}\right): \delta 284.4\left(\mathrm{dd}, \mathrm{C} 6, \mathrm{~J}_{\mathrm{P}-\mathrm{C}}=6.3,19.9 \mathrm{~Hz}\right), 217.0\left(\mathrm{dd}, \mathrm{CO} 3, \mathrm{~J}_{\mathrm{P}-\mathrm{C}}=\right.$ 7.2, $23.8 \mathrm{~Hz}), 208.2\left(\mathrm{dd}, \mathrm{CO} 2, \mathrm{~J}_{\mathrm{P}-\mathrm{C}}=5.9,6.7 \mathrm{~Hz}\right), 163.8(\mathrm{C} 7), 134.9$ (C10), 127.9 (C8), $127.6(\mathrm{C} 9), 138.7\left(\mathrm{~d}, \mathrm{P} 1 \mathrm{PhC}, \mathrm{J}_{\mathrm{P}-\mathrm{C}}=35.3 \mathrm{~Hz}\right), 137.1\left(\mathrm{dd}, \mathrm{P} 2 \mathrm{PhC}, \mathrm{J}_{\mathrm{P}-\mathrm{C}}=34.7,2.5\right.$ $\mathrm{Hz}), 133.0\left(\mathrm{~d}, \mathrm{P} 1 \mathrm{PhC}\right.$ ortho $\left._{\text {o }}, \mathrm{J}_{\mathrm{P}-\mathrm{C}}=11.7 \mathrm{~Hz}\right), 132.4\left(\mathrm{~d}, \mathrm{P} 2 \mathrm{PhC}_{\text {ortho }}, \mathrm{J}_{\mathrm{P}-\mathrm{C}}=11.2 \mathrm{~Hz}\right), 129.2$ $\left(\mathrm{P} 1 \mathrm{PhC}_{\text {meta }}\right), 129.1$ ( $\left.\mathrm{P} 2 \mathrm{PhC}_{\text {meta }}\right), 130.6\left(\mathrm{P} 1 \mathrm{PhC}_{\text {para }}\right), 130.1\left(\mathrm{P} \mathrm{Ph}_{\text {para }}\right), 75.2(\mathrm{C} 11), 15.4$ (C12), 33.0 (dd, P-C13H $2, \mathrm{~J}_{\mathrm{P}-\mathrm{C}}=28.1,17.6 \mathrm{~Hz}$ ), $30.7\left(\mathrm{dd}, \mathrm{P}-\mathbf{C 1 4 H}_{2}, \mathrm{~J}_{\mathrm{P}-\mathrm{C}}=24.3,13.6\right.$ $\mathrm{Hz}) .-{ }^{31} \mathrm{P}$ NMR $\left(\mathrm{CD}_{2} \mathrm{Cl}_{2}, \mathrm{ppm}\right): \delta 42.26\left(\mathrm{dd}, \mathbf{P} 2 \mathrm{Ph}_{2}, \mathrm{~J}_{\mathrm{P}-\mathrm{W}}=223.1 \mathrm{~Hz}, \mathrm{~J}_{\mathrm{P}-\mathrm{P}}=7.8 \mathrm{~Hz}\right)$, $41.18\left(\mathrm{dd}, \mathbf{P}_{\mathrm{PPh}}, \mathrm{J}_{\mathrm{P}-\mathrm{W}}=181.7 \mathrm{~Hz}, \mathrm{~J}_{\mathrm{P}-\mathrm{P}}=7.8 \mathrm{~Hz}\right) .-\mathrm{IR}\left(\mathrm{KBr}, \mathrm{cm}^{-1}\right): v_{\mathrm{CO}}=1966(\mathrm{~s})$, 1866 (s), 1846 (s). Anal. Calcd for $\mathrm{C}_{36} \mathrm{H}_{32} \mathrm{O}_{4} \mathrm{P}_{2} \mathrm{SW}$ : C, 53.66; H, 4.00. Found: C, 53.38; H, 4.12.

6: Yield: $0.378 \mathrm{~g}, 0.478 \mathrm{mmol}, 40.2 \%$, black crystals

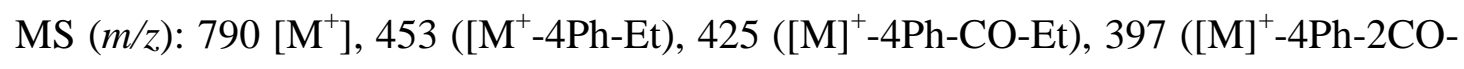
Et). - ${ }^{1} \mathrm{H}$ NMR $\left(\mathrm{CD}_{2} \mathrm{Cl}_{2}, \mathrm{ppm}\right): \delta 7.11$ (dd, H10, 1.7, $\left.0.8 \mathrm{~Hz}\right), 6.65$ (dd, H8, 3.5, 0.8 Hz), 6.30 (dd, H9, 3.5, 1.7 Hz), 7.28-7.46, 7.59-7.73 (m, PPh $), 4.51$ (q, H1 1, 7.0 Hz), 1.35 (t, H12, $7.0 \mathrm{~Hz}$ ), 2.47-2.80 (m, P-CHz). - ${ }^{13} \mathrm{C} \mathrm{NMR}\left(\mathrm{CD}_{2} \mathrm{Cl}_{2}, \mathrm{ppm}\right): \delta 279.1$ (dd, $\left.\mathrm{C} 6, \mathrm{~J}_{\mathrm{P}-\mathrm{C}}=6.2,18.8 \mathrm{~Hz}\right), 217.8\left(\mathrm{dd}, \mathrm{CO} 3, \mathrm{~J}_{\mathrm{P}-\mathrm{C}}=7.2,24.1 \mathrm{~Hz}\right), 208.1\left(\mathrm{~d}, \mathrm{CO} 2, \mathrm{~J}_{\mathrm{P}-\mathrm{C}}=\right.$ $6.3 \mathrm{~Hz}), 170.0$ (C7), 144.9 (C10), 112.3 (C8), 107.3 (C9), 138.5 (dd, P1PhC, J $\mathrm{P}_{-\mathrm{C}}=$ 34.8, 2.1 Hz), $137.3\left(\mathrm{dd}, \mathrm{P} 2 \mathrm{PhC}, \mathrm{J}_{\mathrm{P}-\mathrm{C}}=35.0,2.5 \mathrm{~Hz}\right), 132.7\left(\mathrm{~d}, \mathrm{P} 1 \mathrm{PhC}\right.$ ortho, $\mathrm{J}_{\mathrm{P}-\mathrm{C}}=11.2$ $\mathrm{Hz}), 132.2\left(\mathrm{~d}, \mathrm{P} 2 \mathrm{PhC}_{\text {ortho }}, \mathrm{J}_{\mathrm{P}-\mathrm{C}}=11.2 \mathrm{~Hz}\right), 129.0\left(\mathrm{~d}, \mathrm{P} 1 \mathrm{PhC} \mathrm{meta}_{\text {ma }}, \mathrm{J}_{\mathrm{P}-\mathrm{C}}=10.8 \mathrm{~Hz}\right), 128.8$ $\left(\mathrm{d}, \mathrm{P} 2 \mathrm{PhC}_{\text {meta }}, \mathrm{J}_{\mathrm{P}-\mathrm{C}}=9.1 \mathrm{~Hz}\right), 130.2(\mathrm{P} 1 \mathrm{PhC}$ para $), 129.9\left(\mathrm{~d}, \mathrm{P} 2 \mathrm{PhC}\right.$ para, $\left.\mathrm{J}_{\mathrm{P}-\mathrm{C}}=1.7 \mathrm{~Hz}\right)$, 74.6 (C11), 15.5 (C12), $32.5\left(\mathrm{dd}, \mathbf{C 1 3 H}_{2}, \mathrm{~J}_{\mathrm{P}-\mathrm{C}}=28.1,17.6 \mathrm{~Hz}\right), 30.1\left(\mathrm{dd}, \mathbf{C} 14 \mathrm{H}_{2}, \mathrm{~J}_{\mathrm{P}-\mathrm{C}}\right.$ $=24.6,14.0 \mathrm{~Hz}) .-{ }^{31} \mathrm{P}$ NMR $\left(\mathrm{CD}_{2} \mathrm{Cl}_{2}, \mathrm{ppm}\right): \delta 41.66\left(\mathrm{dd}, \mathbf{P} 2 \mathrm{Ph}_{2}, \mathrm{~J}_{\mathrm{P}-\mathrm{W}}=224.2 \mathrm{~Hz}, \mathrm{~J}_{\mathrm{P}-\mathrm{P}}\right.$ $=7.7 \mathrm{~Hz}), 40.51\left(\mathrm{dd}, \mathbf{P 1 P h}_{2}, \mathrm{~J}_{\mathrm{P}-\mathrm{W}}=181.8 \mathrm{~Hz}, \mathrm{~J}_{\mathrm{P}-\mathrm{P}}=7.9 \mathrm{~Hz}\right) .-\mathrm{IR}\left(\mathrm{KBr}, \mathrm{cm}^{-1}\right): v_{\mathrm{CO}}=$ 1962 (s), 1866 (s), 1841 (s). Anal. Calcd for $\mathrm{C}_{36} \mathrm{H}_{32} \mathrm{O}_{5} \mathrm{P}_{2} \mathrm{~W}: \mathrm{C}, 54.70$; H, 4.08. Found: C, 54.32; H, 4.10.

E: White crystals

${ }^{1} \mathrm{H}$ NMR $\left(\mathrm{CDCl}_{3}, \mathrm{ppm}\right)$ : 7.30 -7.44, 7.49-7.61 (m, PPh $), 2.50-2.68\left(\mathrm{~m}, \mathrm{PCH}_{2}\right) .-{ }^{13} \mathrm{C}$ $\mathrm{NMR}\left(\mathrm{CDCl}_{3}, \mathrm{ppm}\right): \delta 208.2\left(\mathrm{dd}, \mathrm{CO} 3, \mathrm{~J}_{\mathrm{P}-\mathrm{C}}=7.1,23.1 \mathrm{~Hz}\right), 201.3\left(\mathrm{dd}, \mathrm{CO} 2, \mathrm{~J}_{\mathrm{P}-\mathrm{C}}=\right.$ 6.8, 6.4 Hz), 136.1 (d, P1PhC, $\left.\mathrm{J}_{\mathrm{P}-\mathrm{C}}=39.7 \mathrm{~Hz}\right), 131.6\left(\mathrm{~d}, \mathrm{P} 1 \mathrm{PhC} \mathrm{C}_{\text {ortho }}, \mathrm{J}_{\mathrm{P}-\mathrm{C}}=10.0 \mathrm{~Hz}\right)$, $128.9\left(\mathrm{P} 1 \mathrm{PhC}_{\text {meta }}, \mathrm{J}_{\mathrm{P}-\mathrm{C}}=9.7 \mathrm{~Hz}\right), 130.0\left(\mathrm{P} 1 \mathrm{PhC}_{\text {para }}\right), 29.8\left(\mathrm{dd}, \mathrm{P}_{-} \mathrm{CH}_{2}, \mathrm{~J}_{\mathrm{P}-\mathrm{C}}=29.8,13.6\right.$ $\mathrm{Hz})$. - ${ }^{31} \mathrm{P}$ NMR $\left(\mathrm{CD}_{2} \mathrm{Cl}_{2}, \mathrm{ppm}\right): \delta 40.70\left(\mathrm{dd}, \mathbf{P P h}_{2}, \mathrm{~J}_{\mathrm{P}-\mathrm{W}}=230.4 \mathrm{~Hz}, \mathrm{~J}_{\mathrm{P}-\mathrm{P}}=5.5 \mathrm{~Hz}\right) .-$ IR $\left(\mathrm{KBr}, \mathrm{cm}^{-1}\right): v_{\mathrm{CO}}=2013(\mathrm{~s}), 1920(\mathrm{~s}), 1886(\mathrm{~s}), 1872$ (s). 


\subsubsection{Synthesis of mer-[W(dppe $\left.)(\mathrm{CO})_{3}\left\{\mathrm{C}(\mathrm{CHA}) \mathrm{C}_{4} \mathrm{H}_{4} \mathrm{~S}\right\}\right], \quad(7)$, and mer- $\left[\mathrm{W}(\mathrm{dppe})(\mathrm{CO})_{3}\left\{\mathrm{C}(\mathrm{CHA}) \mathrm{C}_{4} \mathrm{H}_{4} \mathrm{O}\right\}\right],(8)$}

Complex C (0.260 g, $0.503 \mathrm{mmol})$ and dppe $(0.249 \mathrm{~g}, 0.625 \mathrm{mmol})$ or complex D $(0.375 \mathrm{~g}, 0.748 \mathrm{mmol})$ and dppe $(0.305 \mathrm{~g}, 0.766 \mathrm{mmol})$ was dissolved in $20 \mathrm{~mL}$ of toluene, and allowed to reflux with stirring for 24 hours. The reaction mixture was initially bright yellow and gradually became a dark wine red solution. TLC indicated the presence of both the fac and mer isomers. The solvent was removed, yielding a dark red solid as the crude product. The crude product was thus purified on a silica gel column using hexane:DCM gradient elution. Two bands were collected, namely, the starting aminocarbene, $\mathbf{C}$ or $\mathbf{D}$, and the target complex, 7 or $\mathbf{8}$. Due to extremely low yields, the percentage yields could only be estimated.

7: Yield: $\pm 15 \%$, orange solid

${ }^{1} \mathrm{H}$ NMR $\left(\mathrm{CD}_{2} \mathrm{Cl}_{2}, \mathrm{ppm}\right): \delta 8.40$ (s (b), N-H), 7.02-7.45 (m, H10), 6.91 (dd, H9, 3.6, $4.9 \mathrm{~Hz}$ ), 6.52 (dd, H8, 3.5, 1.2 Hz), 7.02 -7.45, 7.57-7.72 (m, PPh ), 3.90-4.05 (m,

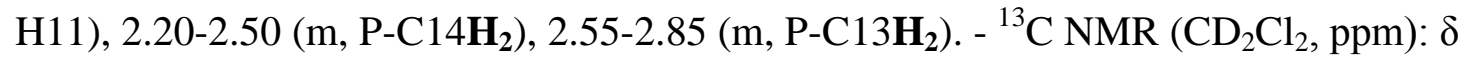
216.9 (CO3), 208.7 (CO2), 159.5 (C7), 126.5 (C10), 121.0 (C8), 113.7 (C9), 140.1 (d, $\left.\mathrm{P} 1 \mathrm{PhC}, \mathrm{J}_{\mathrm{P}-\mathrm{C}}=34.7 \mathrm{~Hz}\right), 136.8\left(\mathrm{~d}, \mathrm{P} 2 \mathrm{PhC}, \mathrm{J}_{\mathrm{P}-\mathrm{C}}=39.2 \mathrm{~Hz}\right), 132.2\left(\mathrm{P}_{\mathrm{PhC}} \mathrm{Prtho}\right), 132.1$ $\left(\mathrm{P} 2 \mathrm{PhC}_{\text {ortho }}\right), 128.8 \quad\left(\mathrm{P} \mathrm{PhC}_{\text {meta }}\right), 128.7 \quad\left(\mathrm{P} 2 \mathrm{PhC}_{\text {meta }}\right), 130.6 \quad\left(\mathrm{P} \mathrm{PhC}_{\text {para }}\right), 130.6$ $\left(\mathrm{P} 2 \mathrm{PhC}_{\text {para }}\right)$, 58.7, 32.0, 25.3, 24.4 (Cyclohexyl-NH), $33.4\left(\mathrm{P}_{-\mathrm{C13H}}\right), 30.9$ (PC14H $\left.H_{2}\right) .{ }^{31} \mathrm{P}$ NMR $\left(\mathrm{CD}_{2} \mathrm{Cl}_{2}, \mathrm{ppm}\right): \delta 45.56\left(\mathrm{~d}, \mathbf{P} 2 \mathrm{Ph}_{2}, \mathrm{~J}_{\mathrm{P}-\mathrm{P}}=11.7 \mathrm{~Hz}\right), 40.18(\mathrm{~d}$, $\left.\mathbf{P} 1 \mathrm{Ph}_{2}, \mathrm{~J}_{\mathrm{P}-\mathrm{P}}=11.6 \mathrm{~Hz}\right)$. - IR $\left(\mathrm{KBr}, \mathrm{cm}^{-1}\right): v_{\mathrm{CO}}=1916$ (s), 1829 (s). Anal. Calcd for $\mathrm{C}_{40} \mathrm{H}_{39} \mathrm{NO}_{3} \mathrm{P}_{2} \mathrm{SW}$ : C, 55.89; H, 4.57; N, 1.63. Found: C, 56.00; H, 4.77; N, 1.52.

8: Yield: $\pm 10 \%$, orange solid

MS $(m / z): 843[\mathrm{M}]^{+}, 453\left([\mathrm{M}]^{+}-4 \mathrm{Ph}-\mathrm{CO}-C y c l o h e x y l\right), 425 \quad\left([\mathrm{M}]^{+}-4 \mathrm{Ph}-2 \mathrm{CO}-\right.$ Cyclohexyl), 397 ([M] $\left.{ }^{+}-4 \mathrm{Ph}-3 \mathrm{CO}-\mathrm{Cyclohexyl}\right) .{ }^{1} \mathrm{H}$ NMR $\left(\mathrm{CD}_{2} \mathrm{Cl}_{2}, \mathrm{ppm}\right): \delta 8.59$ (s (b), N-H), 7.03 (s, H10), 6.65 (s, H8), 6.26 (dd, H9, 3.2, 1.5 Hz), 7.25-7.45, 7.55-7.75 (m, PPh $)$, 4.15-4.30 (m, H11), 2.220-2.50 (m, P-CH2). $\left.{ }^{13} \mathrm{C} \mathrm{NMR} \mathrm{(CD}{ }_{3} \mathrm{CN}, \mathrm{ppm}\right): \delta$ 218.5 (CO3), 202.9 (CO2), 161.7 (C7), 145.2 (C10), 113.5 (C8), 108.3 (C9), 131.2$139.2(\mathrm{~m}, \mathrm{Ph})$, 55.7, 34.0, 26.2, 25.6 (Cyclohexyl-NH), $35.7\left(\mathrm{P}-\mathbf{C 1 3 H} \mathrm{H}_{2}\right), 32.6(\mathrm{P}-$ C14H $\left.\mathrm{H}_{2}\right)$. - ${ }^{31} \mathrm{P}$ NMR $\left(\mathrm{CD}_{2} \mathrm{Cl}_{2}, \mathrm{ppm}\right): \delta 46.36\left(\mathrm{dd}, \mathbf{P} \mathrm{Ph}_{2}, \mathrm{~J}_{\mathrm{P}-\mathrm{P}}=13.6 \mathrm{~Hz}, \mathrm{~J}_{\mathrm{P}-\mathrm{W}}=222.9\right.$ $\mathrm{Hz}), 39.08$ (d, $\mathbf{P 1 P h}$, J $\left.\mathrm{J}_{\mathrm{P} P}=13.4 \mathrm{~Hz}\right)$. IR $\left(\mathrm{KBr}, \mathrm{cm}^{-1}\right): v_{\mathrm{CO}}=1914$ (s), 1821 (s). Anal. 
Calcd for $\mathrm{C}_{40} \mathrm{H}_{39} \mathrm{NO}_{4} \mathrm{P}_{2} \mathrm{~W}$ : C, 56.96; H, 4.66; N, 1.66. Found: C, 56.66; H, 4.51; N, 1.55 .

\subsubsection{Synthesis of $c$ is $-\left[\mathrm{W}\left(\mathrm{P}(\mathrm{OPh})_{3}\right)(\mathrm{CO})_{4}\left\{\mathrm{C}(\mathrm{OEt}) \mathrm{C}_{4} \mathrm{H}_{4} \mathrm{~S}\right\}\right]$, (9cis $)$}

Complex A $(0.464 \mathrm{~g}, 1.00 \mathrm{mmol})$ and $\mathrm{P}(\mathrm{OPh})_{3}(0.465 \mathrm{~g}, 1.50 \mathrm{mmol})$ were dissolved in $30 \mathrm{~mL}$ of toluene (wine-red solution). This mixture was left to reflux overnight (dark brown solution), and the solvent was removed (dark brown solid). The crude product was purified on silica gel using hexane:DCM gradients. Unreacted carbene A $(0.100 \mathrm{~g}, 0.215 \mathrm{mmol}, 21.5 \%)$ and two products were obtained according to TLC. The two products were identified as the cis and trans isomers of the product. The trans product, 9trans, is orange-brown in solution and elutes after the starting carbene A. This product was an intractable mixture of compounds and could not be characterized unambiguously. The cis product, 9cis, eluted directly after the trans isomer as a dark red-brown solution.

9cis: Yield: $0.167 \mathrm{~g}, 0.223 \mathrm{mmol}, 22.3 \%$, reddish-brown oil

- ${ }^{1} \mathrm{H}$ NMR ( $\left.\mathrm{CDCl}_{3}, \mathrm{ppm}\right): \delta 7.81$ (dd, H8, 3.9, $\left.1.3 \mathrm{~Hz}\right), 7.65$ (dd, H10, 5.0, $1.0 \mathrm{~Hz}$ ), 6.91 (d, H9, 1.3 Hz), 7.04-7.33 (m, POPh $), 4.42$ (q, H11, 7.1 Hz), 1.27 (t, H12, 7.0 Hz). - ${ }^{13} \mathrm{C} \mathrm{NMR}\left(\mathrm{CDCl}_{3}, \mathrm{ppm}\right): \delta 295.8$ (C6), 209.4 (d, CO1, J $\left.\mathrm{P}_{\mathrm{C}}=5.6 \mathrm{~Hz}\right), 206.3$ (d, $\left.\mathrm{CO} 3, \mathrm{~J}_{\mathrm{P}-\mathrm{C}}=7.3 \mathrm{~Hz}\right), 202.6\left(\mathrm{~d}, \mathrm{CO} 2, \mathrm{~J}_{\mathrm{P}-\mathrm{C}}=7.6 \mathrm{~Hz}\right), 159.9(\mathrm{C} 7), 139.2(\mathrm{C} 8), 134.7$ (C10), 128.9 (C9), 151.6 (OPhC), $129.4\left(\mathrm{PhC}_{\text {ortho }}\right), 121.2\left(\mathrm{PhC}_{\text {para }}\right), 129.4$ (d, $\left.\mathrm{PhC}_{\text {meta }}\right), 77.2$ (C11), 14.1 (C12). - ${ }^{31} \mathrm{P}$ NMR $\left(\mathrm{CDCl}_{3}, \mathrm{ppm}\right): \delta 133.8\left(\mathbf{P O P h}_{3}\right)$. - IR $\left(\right.$ Hexane, $\left.\mathrm{cm}^{-1}\right): v_{\mathrm{CO}}=1996(\mathrm{~s}), 1917(\mathrm{~s}), 1889(\mathrm{~s})$.

\subsubsection{Synthesis of cis $-\left[\mathrm{W}\left(\mathrm{P}(\mathrm{OPh})_{3}\right)(\mathrm{CO})_{4}\left\{\mathrm{C}(\mathrm{OEt}) \mathrm{C}_{4} \mathrm{H}_{4} \mathrm{O}\right\}\right]$, (10cis $)$}

Complex B $(0.448 \mathrm{~g}, 1.00 \mathrm{mmol})$ and $\mathrm{P}(\mathrm{OPh})_{3}(0.465 \mathrm{~g}, 1.50 \mathrm{mmol})$ were dissolved in $40 \mathrm{~mL}$ of toluene (wine red solution) and left to reflux for 24 hours (brown solution). The solvent was then removed, yielding a brown solid as the crude product. TLC indicated little starting monocarbene $\mathbf{B}$ and two products, cis and trans isomers, were present. Starting monocarbene $\mathbf{B}$ was removed from the products on a silica gel filter using hexane:DCM gradient elution. The isomers were then separated on silica gel column using hexane:DCM gradient elution. Elution patterns and colours for the isomers were the same as for the thiophene analogues. Again, the trans product was an intractable mixture of compounds and could not be characterized unambiguously. 
10cis: Yield: $0.138 \mathrm{~g}, 0.189 \mathrm{mmol}, 18.9 \%$, red-brown oil

- ${ }^{1} \mathrm{H}$ NMR (CDCl 3 , ppm): $\delta 7.60$ (dd, H10, 5.2, $\left.1.3 \mathrm{~Hz}\right), 6.95$ (m, H8), 6.46 (dd, H9, 3.4, 1.6 Hz), 7.13-7.46 (m, POPh 3 ), 4.28 (q, H11, 7.1 Hz), 1.36 (m, H12,). - ${ }^{13} \mathrm{C}$ NMR $\left(\mathrm{CDCl}_{3}, \mathrm{ppm}\right): \delta 288.7\left(\mathrm{~d}, \mathrm{C} 6, \mathrm{~J}_{\mathrm{P}-\mathrm{C}}=8.9 \mathrm{~Hz}\right), 210.9\left(\mathrm{~d}, \mathrm{CO} 1, \mathrm{~J}_{\mathrm{P}-\mathrm{C}}=10.7 \mathrm{~Hz}\right), 207.3$ $\left(\mathrm{d}, \mathrm{CO} 3, \mathrm{~J}_{\mathrm{P}-\mathrm{C}}=7.5 \mathrm{~Hz}\right), 203.6\left(\mathrm{~d}, \mathrm{CO} 2, \mathrm{~J}_{\mathrm{P}-\mathrm{C}}=6.8 \mathrm{~Hz}\right), 166.7(\mathrm{C} 7), 151.7(\mathrm{C} 10), 115.3$ (C8), 112.3 (C9), 155.5 (OPhC), $121.2\left(\mathrm{PhC}_{\text {ortho }}\right), 124.1\left(\mathrm{PhC}_{\text {para }}\right), 129.3\left(\mathrm{PhC}_{\text {meta }}\right)$, 77.2 (C11), 14.1 (C12). - ${ }^{31} \mathrm{P}$ NMR $\left(\mathrm{CDCl}_{3}, \mathrm{ppm}\right): \delta 134.7\left(\mathrm{POPh}_{3}\right)$. - IR $($ Hexane, $\left.\mathrm{cm}^{-1}\right): v_{\mathrm{CO}}=2000(\mathrm{~s}), 1918(\mathrm{~s}), 1893(\mathrm{~s})$.

\subsubsection{Synthesis of cis- and trans-[W(P(OPh $\left.\left.)_{3}\right)(\mathrm{CO})_{4}\left\{\mathrm{C}(\mathrm{CHA}) \mathrm{C}_{4} \mathrm{H}_{4} \mathrm{~S}\right\}\right]$, (11cis) and (11trans)}

Complex C $(0.516 \mathrm{~g}, 1.00 \mathrm{mmol})$ and $\mathrm{P}(\mathrm{OPh})_{3}(0.465 \mathrm{~g}, 1.50 \mathrm{mmol})$ were dissolved in $40 \mathrm{~mL}$ of toluene, resulting in a bright yellow solution. The reaction mixture was allowed to reflux, with stirring for 6 hours. At this point, most of the starting material had and thus the solution was cooled and the solvent removed. A bright orange solid was obtained. The crude product was then purified on a silica gel column using hexane:DCM gradient elution. Two fractions were collected. The first fraction was a bright orange product 11trans. The second fraction was bright orange-brown and contained the product $\mathbf{1 1 C i s}$. The products were obtained as orange oils.

11cis: Yield: $0.242 \mathrm{~g}, 0.302 \mathrm{mmol}, 30.2 \%$, brown-orange oil

${ }^{1} \mathrm{H}$ NMR $\left(\mathrm{CDCl}_{3}, \mathrm{ppm}\right): \delta 8.20$ (s (b), N-H), 7.39 (dd, H10, 3.7, 1.2 Hz), 7.12 (dd, H8, 3.7, $1.2 \mathrm{~Hz}$ ), 6.88 (m, H9), 7.01-7.33 (m, POPh $)$, 4.04-4.15 (m, H11), 1.05-1.29, 1.34-1.71 (m, Cyclohexyl-NH). - ${ }^{13} \mathrm{C}$ NMR $\left(\mathrm{CDCl}_{3}, \mathrm{ppm}\right): \delta 247.5\left(\mathrm{~d}, \mathrm{C} 6, \mathrm{~J}_{\mathrm{P}-\mathrm{C}}=11.0\right.$ $\mathrm{Hz}), 209.3\left(\mathrm{~d}, \mathrm{CO} 1, \mathrm{~J}_{\mathrm{P}-\mathrm{C}}=8.9 \mathrm{~Hz}\right), 206.8\left(\mathrm{~d}, \mathrm{CO} 3, \mathrm{~J}_{\mathrm{P}-\mathrm{C}}=6.5 \mathrm{~Hz}\right), 203.1\left(\mathrm{~d}, \mathrm{CO} 2, \mathrm{~J}_{\mathrm{P}-\mathrm{C}}\right.$ = $10.8 \mathrm{~Hz}$ ), 158.6 (C7), 128.3 (C10), 126.7 (C8), 126.4 (C9), 152.1 (OPhC), 121.5 $\left(\mathrm{PhC}_{\text {ortho }}\right), 124.1$ (s, $\left.\mathrm{PhC}_{\text {para }}\right), 129.4\left(\mathrm{PhC}_{\text {meta }}\right), 62.2,32.7,24.4,24.2$ (CyclohexylNH). - ${ }^{31} \mathrm{P} \mathrm{NMR}\left(\mathrm{CDCl}_{3}, \mathrm{ppm}\right): \delta 132.9\left(\mathrm{POPh}_{3}\right) .-$ IR $\left(\right.$ Hexane, $\left.\mathrm{cm}^{-1}\right): v_{\mathrm{CO}}=1993(\mathrm{~s})$, 1979(s), 1902 (s), 1871 (s).

11trans: Yield: $0.311 \mathrm{~g}, 0.389 \mathrm{mmol}, 38.9 \%$, bright orange oil

${ }^{1} \mathrm{H}$ NMR $\left(\mathrm{CDCl}_{3}, \mathrm{ppm}\right): \delta 8.18$ (s (b), N-H), $\delta 7.40$ (s, H10), 7.09 (dd, H8, 5.5, 1.7 Hz), 6.87 (m), 7.14-7.42 (m, POPh $)$, 4.02-4.17 (m, H11), 1.06-1.39, 1.55-1.95 (m, Cyclohexyl-NH). - ${ }^{13} \mathrm{C} \mathrm{NMR}\left(\mathrm{CDCl}_{3}, \mathrm{ppm}\right): \delta 241.2\left(\mathrm{~d}, \mathrm{C} 6, \mathrm{~J}_{\mathrm{P}-\mathrm{C}}=10.9 \mathrm{~Hz}\right), 206.2(\mathrm{~d}$, $\left.\mathrm{CO}, \mathrm{J}_{\mathrm{P}-\mathrm{C}}=8.6 \mathrm{~Hz}\right), 152.4(\mathrm{C} 7), 128.0(\mathrm{C} 10), 127.7(\mathrm{C} 8), 127.0(\mathrm{C} 9), 151.6(\mathrm{OPhC})$, 
121,4( $\left.\mathrm{PhC}_{\text {ortho }}\right), 124.7\left(\mathrm{PhC}_{\text {para }}\right), 129.7\left(\mathrm{PhC}_{\text {meta }}\right)$, 64.0, 33.5, 25.1, 25.7 (Cyclohexyl-

$\mathrm{NH}) .-{ }^{31} \mathrm{P} \mathrm{NMR}\left(\mathrm{CDCl}_{3}, \mathrm{ppm}\right): \delta 137.9\left(\mathrm{POPh}_{3}\right) .-$ IR $\left(\right.$ Hexane, $\left.\mathrm{cm}^{-1}\right): v_{\mathrm{CO}}=2024(\mathrm{~s})$, 1935 (vs), 1911 (vs).

2.2.10 Synthesis of cis- and trans-[W(P(OPh $\left.\left.)_{3}\right)(\mathrm{CO})_{4}\left\{\mathrm{C}(\mathrm{CHA}) \mathrm{C}_{4} \mathrm{H}_{4} \mathrm{O}\right\}\right],(12$ cis $)$ and (12trans)

Complex D $(0.502 \mathrm{~g}, 1.00 \mathrm{mmol})$ and $\mathrm{P}(\mathrm{OPh})_{3}(0.465 \mathrm{~g}, 1.50 \mathrm{mmol})$ were dissolved in $40 \mathrm{~mL}$ of toluene, resulting in a bright yellow solution. The reaction mixture was allowed to reflux, with stirring, for 4 hours. A bright orange solution was obtained. The crude product was then purified on a silica gel column using hexane:DCM gradient elution. Two fractions were collected. The first fraction was identified as 12cis, while the second fraction contained the product, 12trans. The products were obtained as orange oils.

12cis: Yield: $0.244 \mathrm{~g}, 0.311 \mathrm{mmol}, 31.1 \%$, orange oil

${ }^{1} \mathrm{H} \mathrm{NMR}\left(\mathrm{CDCl}_{3}, \mathrm{ppm}\right): \delta 8.61$ (s (b), N-H), 7.35 (d, H10, 1.7), 6.81 (d, H8, 3.6), 6.37 (dd, H9, 3.6, 1.8 Hz), 7.00-7.46 (m, POPh $)$ ), 4.00-4.29 (m, H11), 1.10-1.28, 1.39-1.74 (m, Cyclohexyl-NH). - ${ }^{13} \mathrm{C}$ NMR $\left(\mathrm{CDCl}_{3}, \mathrm{ppm}\right): \delta 229.2\left(\mathrm{~d}, \mathrm{C} 6, \mathrm{~J}_{\mathrm{P}-\mathrm{C}}=4.6 \mathrm{~Hz}\right), 210.1$ $\left(\mathrm{d}, \mathrm{CO} 1, \mathrm{~J}_{\mathrm{P}-\mathrm{C}}=5.6 \mathrm{~Hz}\right), 207.8\left(\mathrm{~d}, \mathrm{CO} 3, \mathrm{~J}_{\mathrm{P}-\mathrm{C}}=8.6 \mathrm{~Hz}\right), 204.9\left(\mathrm{~d}, \mathrm{CO} 2, \mathrm{~J}_{\mathrm{P}-\mathrm{C}}=6.9 \mathrm{~Hz}\right)$, 160.8 (C7 ), 142.2 (C10), 112.9 (C8), 115.3 (C9), 152.0 (OPhC), 121.3 ( $\mathrm{PhC}_{\text {ortho }}$ ), 123.9 $\left(\mathrm{PhC}_{\text {para }}\right), 129.2\left(\mathrm{PhC}_{\text {meta }}\right), 61.4,32.8,24.3,25.2$ (Cyclohexyl-NH). - ${ }^{31} \mathrm{P}$ NMR $\left(\mathrm{CDCl}_{3}, \mathrm{ppm}\right): \delta 132.3\left(\mathbf{P O P h}_{3}\right)$. - IR $\left(\right.$ Hexane, $\left.\mathrm{cm}^{-1}\right): v_{\mathrm{CO}}=1984$ (s), 1935 (s), 1876 (vs).

12trans: Yield: $0.289 \mathrm{~g}, 0.368 \mathrm{mmol}, 36.8 \%$, bright orange oil

${ }^{1} \mathrm{H}$ NMR $\left(\mathrm{CDCl}_{3}, \mathrm{ppm}\right): \delta 8.54$ (s (b), N-H), $\delta 7.44$ (d, H10, $\left.2.0 \mathrm{~Hz}\right), 6.51$ (dd, H8, 3.7, $1.8 \mathrm{~Hz}$ ), 6.49 (dd, H9, 3.6, 1.8 Hz), 7.00-7.38 (m, POPh $), 4.09-4.23$ (m, H11), 1.11-1.49, 1.62-1.95 (m, Cyclohexyl-NH). ${ }^{13} \mathrm{C} \mathrm{NMR}\left(\mathrm{CDCl}_{3}, \mathrm{ppm}\right): \delta 223.9$ (d, C6, $\left.\mathrm{J}_{\mathrm{P}-\mathrm{C}}=9.7 \mathrm{~Hz}\right), 205.9\left(\mathrm{~d}, \mathrm{CO}, \mathrm{J}_{\mathrm{P}-\mathrm{C}}=9.2 \mathrm{~Hz}\right), 159.7(\mathrm{C} 7), 143.2(\mathrm{C} 10), 113.5(\mathrm{C} 8)$, 113.7 (C9), 151.5 (OPhC), 121.2 ( $\left.\mathrm{PhC}_{\text {ortho }}\right), 124.5$ ( $\left.\mathrm{PhC}_{\text {para }}\right), 129.5\left(\mathrm{PhC}_{\text {meta }}\right), 63.1$, 33.0, 24.4, 25.1 (Cyclohexyl-NH). - ${ }^{31} \mathrm{P}$ NMR $\left(\mathrm{CDCl}_{3}, \mathrm{ppm}\right): \delta 138.2\left(\mathbf{P O P h}_{3}\right)$. - IR (Hexane, $\left.\mathrm{cm}^{-1}\right): v_{\mathrm{CO}}=2020(\mathrm{~s}), 1930(\mathrm{vs}), 1894$ (vs). 


\subsection{Cyclic Voltammetry}

Cyclic voltammogram (CV) and linear sweep voltammogram (LSV) measurements were performed on $0.0005 \mathrm{~mol} \mathrm{dm}{ }^{-3}$ compound solutions in dry acetonitrile containing $\quad 0.1 \mathrm{~mol} \mathrm{dm}^{-3}$ tetra- $n$-butylammonium hexafluorophosphate, $\left(\left[{ }^{\mathrm{n}}\left(\mathrm{Bu}_{4}\right) \mathrm{N}\right]\left[\mathrm{PF}_{6}\right]\right)$, as supporting electrolyte and under a blanket of purified argon at 25 ${ }^{\circ} \mathrm{C}$ utilizing a BAS 100B/W voltammograph. A three-electrode cell, with a glassy carbon (surface area $7.07 \times 10^{-6} \mathrm{~m}^{2}$ ) working electrode, Pt auxiliary electrode and an $\mathrm{Ag}$ wire reference electrode were used [29]. Scan rates were 0.050-5.000 $\mathrm{V} \mathrm{s}^{-1}$. Successive experiments under the same experimental conditions showed that all oxidation and formal reduction potentials were reproducible within $10 \mathrm{mV}$. All cited potentials were referenced against the $\mathrm{FcH} / \mathrm{FcH}^{+}$couple as suggested by IUPAC [30]. Ferrocene $(\mathrm{FcH})$ exhibited a peak separation $\Delta E_{\mathrm{p}}=E_{\mathrm{pa}}-E_{\mathrm{pc}}=0.069 \mathrm{~V}$ and $i_{p d} i_{p a}=$ 1.00 under our experimental conditions. $E_{\mathrm{pa}}\left(E_{\mathrm{pc}}\right)=$ anodic (cathodic) peak potential and $i_{p a}\left(i_{p c}\right)=\operatorname{anodic}\left(\right.$ cathodic) peak current. $E^{\circ \prime}\left(\mathrm{FcH} / \mathrm{FcH}^{+}\right)=0.66(5) \mathrm{V}$ vs SHE in $\left[{ }^{\mathrm{n}}\left(\mathrm{Bu}_{4}\right) \mathrm{N}\right]\left[\mathrm{PF}_{6}\right] / \mathrm{CH}_{3} \mathrm{CN}$ [31]. Decamethyl ferrocene $\left(\mathrm{Fc}^{*},-0.508 \mathrm{~V}\right.$ vs $\left.\mathrm{FcH} / \mathrm{FcH}^{+}\right)$ were used as internal standard.

\subsection{DFT calculations}

Density functional theory (DFT) calculations of this study were performed with the hybrid functional B3LYP [32,33] (and uB3LYP for radical cations or anions) as implemented in the Gaussian 09 program package [34]. Geometries of the neutral and charged complexes were optimized in gas phase with the triple- $\zeta$ basis set 6 $311 \mathrm{G}(\mathrm{d}, \mathrm{p})$ on all atoms except tungsten, where def-TZSVPP [35] was used (this level is denoted $\mathrm{B} 3 \mathrm{LYP} / \mathrm{def} 2-\mathrm{TZSVPP})$. Adiabatic ionization potential and electron affinities were calculated from the energies of the relaxed minimum energy geometries of the charged species. Optimized geometries obtained from DFT calculations were used to perform an NBO analysis by the NBO 3.1 module [36] in Gaussian 09 at the same level of theory.

\section{$2.5 \quad$ X-ray Crystallography}

Single crystal X-ray data for $\mathbf{2}$ cis were collected at $150 \mathrm{~K}$ on a Bruker D8 Venture kappa geometry diffractometer, with duo I $\mu$ s sources, a Photon $100 \mathrm{CMOS}$ detector 
and APEX II [37] control software using Quazar multi-layer optics monochromated, Mo-K $K \alpha$ radiation by means of a combination of $\phi$ and $\omega$ scans. Data for $\mathbf{3}$ were collected at 100(2) K, using a Bruker APEX DUO 4K-CCD diffractometer. Data reduction was performed using SAINT+ [37] and the intensities were corrected for absorption using SADABS [37]. The structures were solved by intrinsic phasing using SHELXTS [38] and refined by full-matrix least squares using SHELXTL and SHELXL-2013 [38]. Data for 5 and E were collected at 180(2) K on a Nonius Kappa CCD diffractometer, using graphite monochromated, Mo K $\alpha$ radiation by means of phi and omega scans, and semi-empirical absorption corrections were based on equivalence. In the structure refinement, all hydrogen atoms were added in calculated positions and treated as riding on the atom to which they are attached. All nonhydrogen atoms were refined with anisotropic displacement parameters, all isotropic displacement parameters for hydrogen atoms were calculated as $\mathrm{X} \times \mathrm{Ueq}$ of the atom to which they are attached, $\mathrm{X}=1.5$ for the methyl hydrogens and 1.2 for all other hydrogens. Data collection, structure solution and refinement details are available in each CIF. Ortep drawings [39] of the four structures are included in Figures 1-4 with ADP's at the 50\% probability level. The crystal structures have been deposited at the Cambridge Crystallographic Data Centre and allocated the deposition numbers: CCDC 955033 (2cis), CCDC 953885 (3), CCDC 953892 (5), CCDC 953895 (E).

\section{Results and Discussion}

\subsection{Synthesis, characterization and X-ray crystallography}

The incorporation of phosphines or phosphites into a Fischer carbene's ligand sphere can either be done before or after the carbene was introduced [14-16,40,41]. If $\mathrm{M}(\mathrm{CO})_{5} \mathrm{PR}_{3}$ is used instead of $\mathrm{M}(\mathrm{CO})_{6}$ during the classical Fischer synthesis, cissubstituted carbenes are formed exclusively [5,14]. A phosphine ligand reduces the electrophilicity of the trans-carbonyl carbon due to the increased back bonding from the metal, making it less available to react with the organolithium reagent. Therefore, the probability of the trans isomer forming, is reduced. If the phosphine is coordinated to the carbene complex by the normal carbonyl substitution methods, i.e. thermal or photolytic substitution, then both the cis and the trans isomers can be formed. Literature concerning bidentate phosphine ligands report carbonyl substitution of the carbene complex as the only means to form the chelated carbene complexes [40]. 
Both $f a c$ and mer isomers can be obtained $[15,17]$. For the purposes of our studies, thermal carbonyl substitution of the monocarbene complex was the preferred method of synthesis.

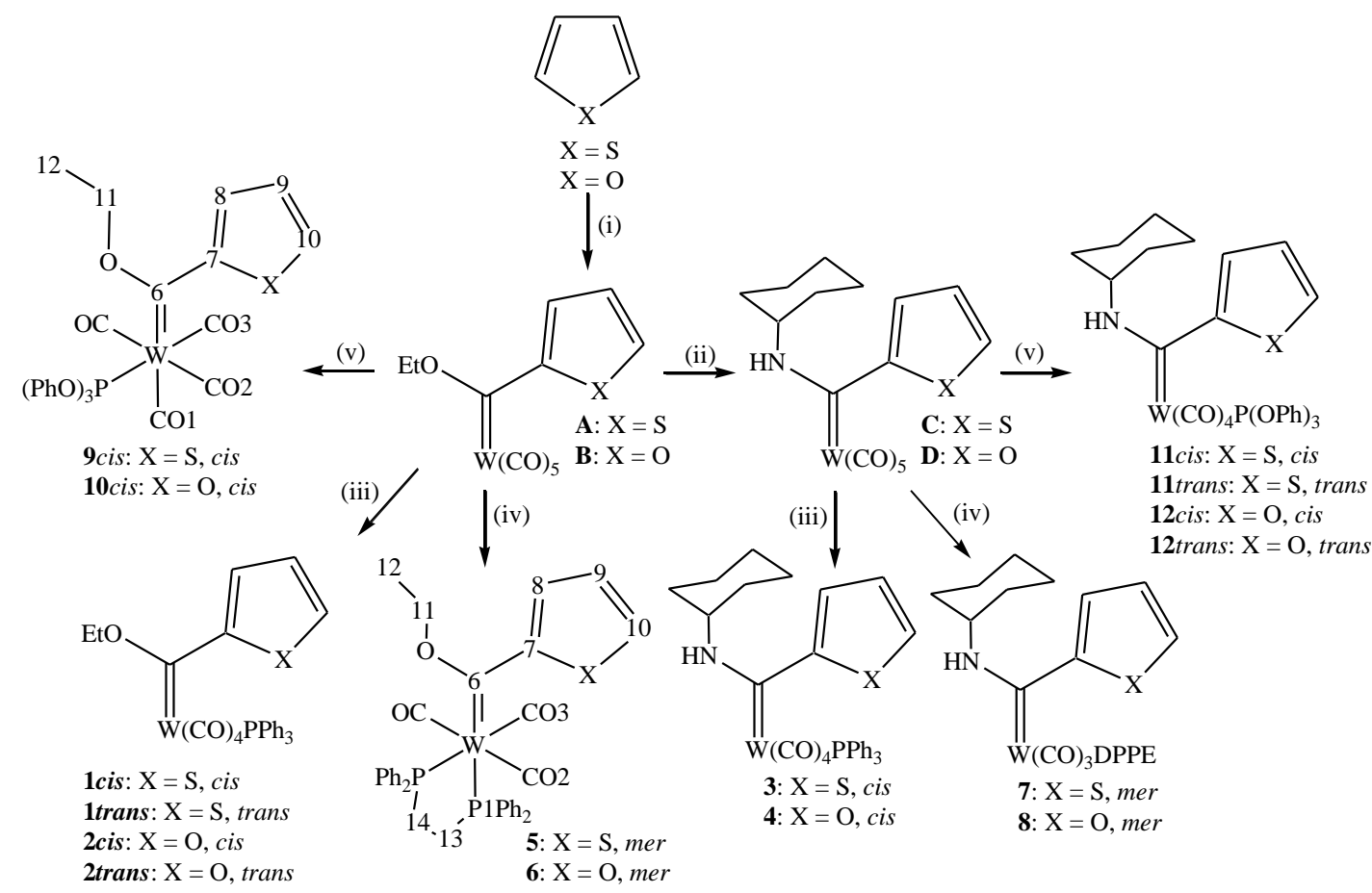

Scheme 1. Reagents and conditions for synthesis of novel isolated complexes: (i)(a) 1 eq. ${ }^{\mathrm{n}} \mathrm{BuLi}, \mathrm{THF}$, $-78{ }^{\circ} \mathrm{C}$; (b) $\left[\mathrm{W}(\mathrm{CO})_{6}\right], \mathrm{THF},-50{ }^{\circ} \mathrm{C}$; (c) 1.1 eq. $\mathrm{Et}_{3} \mathrm{OBF}_{4}$; (ii) 3.3 eq. CHA, THF, rt; (iii) 1.1 eq. $\mathrm{PPh}_{3}$, toluene, reflux; (iv) 1.1 eq. dppe, toluene, reflux; (v) 1.5 eq. $\mathrm{P}(\mathrm{OPh})_{3}$, toluene, reflux.

Monocarbene complexes $\left[(\mathrm{CO})_{5} \mathrm{~W}=\mathrm{C}(\mathrm{OEt}) \mathrm{R}\right]$ with $\mathrm{R}=2$-thienyl (A) or 2-furyl $(\mathbf{B})$ were prepared using well-known Fischer methodology [7,42-44], see Scheme 1 for complex numbering. The phosphorous ligands were introduced into the complex by refluxing the monocarbene complex (A or $\mathbf{B})$ in toluene with an excess of ligand for several hours. For the monosubstituted complexes, the cis isomer was generally obtained in tenfold excess to the trans isomer, according to thin layer chromatography (TLC) analysis. Separation of the cis and trans monophosphine ethoxycarbene isomers of $\mathbf{1}$ and $\mathbf{2}$ proved to be challenging due to the existing isomerisation equilibrium [45]. In fact, Cooke and Fischer [14] found it impossible to separate the isomers from one another. However, the isomers were successfully separated by means of fraction collection. Relatively low yields $(\sim 40 \%)$ were obtained when compared to literature reports for similar phosphine-substituted carbene complexes [46]. In the case of the phosphite complexes, $\mathbf{9}$ and 10, only the cis isomer could be 
characterized as the trans product could not be purified. In the reaction of the starting ethoxycarbene complex (A or B) and the bidentate phosphine ligand, dppe, both the fac and mer isomers (qualitatively in a c.a. 1:3 ratio) were observed on TLC for both complexes 5 and 6. According to literature, both isomers can be obtained but the mer is favoured $[15,17]$, especially at high reaction temperatures. Column chromatography was unsuccessful as the complexes decomposed on the column. Crystallization was thus used for purification. However, only the mer isomer could be isolated with this method and was obtained as black crystals from the reaction mixture. During synthesis of monophosphine carbene complexes, both Werner and Rascher [6] and the Fischer group [47] found several decomposition products that included mono- and diphosphine metal carbonyls. This indicates that both ligand substitution as well as carbene ligand substitution occurred. Similarly, in our reactions, crystals of the known compound $\left[\mathrm{W}(\mathrm{CO})_{4} \mathrm{dppe}\right], \mathbf{E}$ [48-50] were isolated from the crude reaction mixtures of 5 and 6, and identified using XRD. No literature report for the [W(CO) $)_{4}$ dppe] crystal structure could be found, which is thus presented in this work as a novel crystal structure.

The aminocarbene complexes $\mathbf{C}$ and $\mathbf{D}$ were prepared by aminolysis of $\mathbf{A}$ or $\mathbf{B}$ with cyclohexyl amine (CHA). Complexes 3, 4, 7, 8, 11 and 12 were synthesised by thermal substitution of a carbonyl group by $\mathrm{PPh}_{3}(\mathbf{3}, \mathbf{4})$, dppe $(\mathbf{7}, \mathbf{8})$ or $\mathrm{P}(\mathrm{OPh})_{3}(\mathbf{1 1}$, 12) on complex $\mathbf{C}$ or $\mathbf{D}$ (Scheme 1). For the monophosphine derivatives ( $\mathbf{3}$ and $\mathbf{4}$ ), only the cis isomer was obtained. Again, only the mer isomers of complexes $\mathbf{7}$ and $\mathbf{8}$ could by isolated and characterized. Both cis and trans isomers were afforded for the phosphite aminocarbene complexes, $\mathbf{1 1}$ and $\mathbf{1 2 .}$

Complexes 1-12 were characterized using ${ }^{1} \mathrm{H},{ }^{13} \mathrm{C}$ and ${ }^{31} \mathrm{P}$ NMR and infrared spectroscopy as well as mass spectrometry. Atom numbering is shown in Scheme 1. On the ${ }^{1} \mathrm{H}$ NMR spectra, all resonance peaks observed for the heteroaryl ring of the substituted ethoxycarbene derivatives $(\mathbf{1}, \mathbf{2}, \mathbf{5}, \mathbf{6}, 9$ and 10) were shifted more upfield than the corresponding resonance peaks of the two reagent complexes (A and $\mathbf{B})$. This trend is supported by literature data found for similar monophosphines complexes [6]. On the ${ }^{13} \mathrm{C}$ NMR spectra of the aminocarbene complexes, the characteristic upfield shift (50-60ppm) for the carbene carbon (C6) resonance, compared to the 
ethoxycarbene analogues, is evident [51,52]. For the tungsten(0) tetracarbonyl monocarbene complexes, 1-4 and 9-12, a single carbonyl resonance peak was found for the trans isomers, while the cis isomers showed three carbonyl chemical resonances with a relative ratio of $1: 1: 2$, corresponding to three different environments. The chemical shift value for a carbonyl group is dependent on the substituent trans to the carbonyl [53] and the following trend was used to assign the different carbonyl resonances: $\mathrm{CO}<$ phosphite $<$ phosphine, arsine $<$ amine $<$ carbene.

Carbonyl groups can also be distinguished by the difference in magnitude of ${ }^{2} \mathrm{~J}(\mathrm{P}-\mathrm{C})$ coupling constants [54]. A trans carbonyl has a larger ${ }^{2} \mathrm{~J}(\mathrm{P}-\mathrm{C})$ coupling constant (24 $\mathrm{Hz})$ than a cis carbonyl $(6-7 \mathrm{~Hz})$. By contrast, ${ }^{1} \mathrm{~J}(\mathrm{P}-\mathrm{W})$ for cis complexes $(\sim 220 \mathrm{~Hz})$ are larger than for trans complexes $(\sim 205 \mathrm{~Hz})$ [55]. On the ${ }^{31} \mathrm{P}$ spectra of 5-8, two peaks were seen in each case, confirming the mer arrangement of ligands for phosphorous atoms in non-equivalent positions. By comparison with the monophosphine complexes, the assignments for the two phosphine atoms P1 and P2 of the bidentate ligand could be readily made.

Suitable crystals of $\mathbf{2}$ cis, $\mathbf{3}, \mathbf{5}$ and $\mathbf{E}$ were obtained from dichloromethane:hexane (1:1) solutions and molecular structures determined with single-crystal X-ray diffraction analysis (figures 1-4). Selected bond lengths and angles are given in Table 1 of the supplementary material. Due to some disorder in the orientation of the thienyl ring, only the major orientation of the thienyl substituent in $\mathbf{3}$ is shown in Figure 2. For the ethoxy complexes, there is only slight deviation from expected planarity [42], with dihedral angles (O7-C6-C7-S1/O6) of -175.64(12) ${ }^{\circ}(\mathbf{2 c i s})$ and -14.5(4) $)^{\circ}(5)$, while the dihedral angle (N1-C6-C7-S1) for 3 is $146.0(5)^{\circ}$. This large deviation for 3 is ascribed to having a large heteroatom substituent $(\mathrm{CHA})$ and the $\mathrm{PPh}_{3}$ ligand in the same complex. The carbene bond lengths (W1-C6) for the two ethoxy complexes, 2cis and $\mathbf{5}$, are very similar. For the aminocarbene complex, $\mathbf{3}$, this bond length is longer due to decreased $\pi$-backbonding from the metal to the carbene carbon atom [56-59]. Less $\pi$ donation will thus be required from the heteroaryl ring to the carbene carbon atom; therefore, the longer C6-C7 bond length for $\mathbf{3}$ compared to 2 cis and 5. Due to diminished $\pi$-bonding to the carbene carbon atom, the $\mathrm{W}$ metal is more electron-rich and the trans carbonyl bond to the metal is stronger in $\mathbf{3}$. This bond length is 
comparable to the $\mathrm{W} 1-\mathrm{CO} 3$ bond lengths in all three complexes. This observation again correlates with the difference in $\pi$-acceptor abilities of carbonyl, phosphine and carbene ligands.

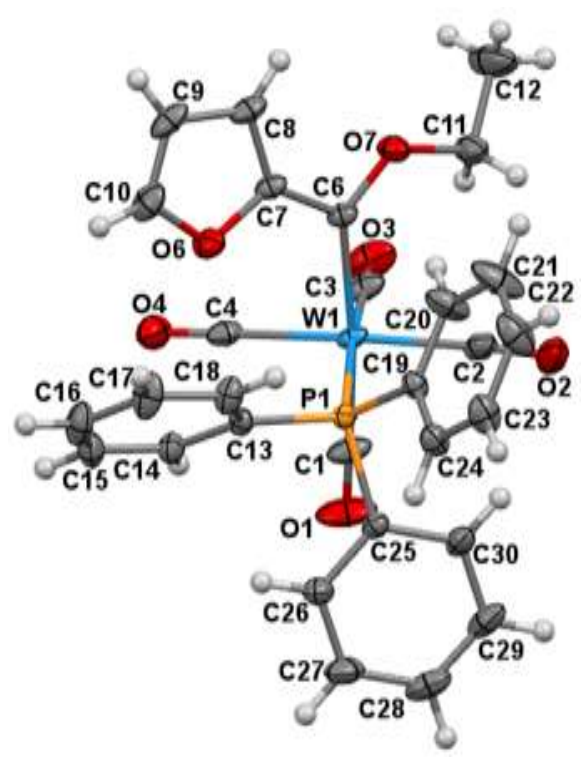

Figure 1. Perspective view of $\mathbf{2}$ cis with thermal ellipsoids drawn at the $50 \%$ probability level

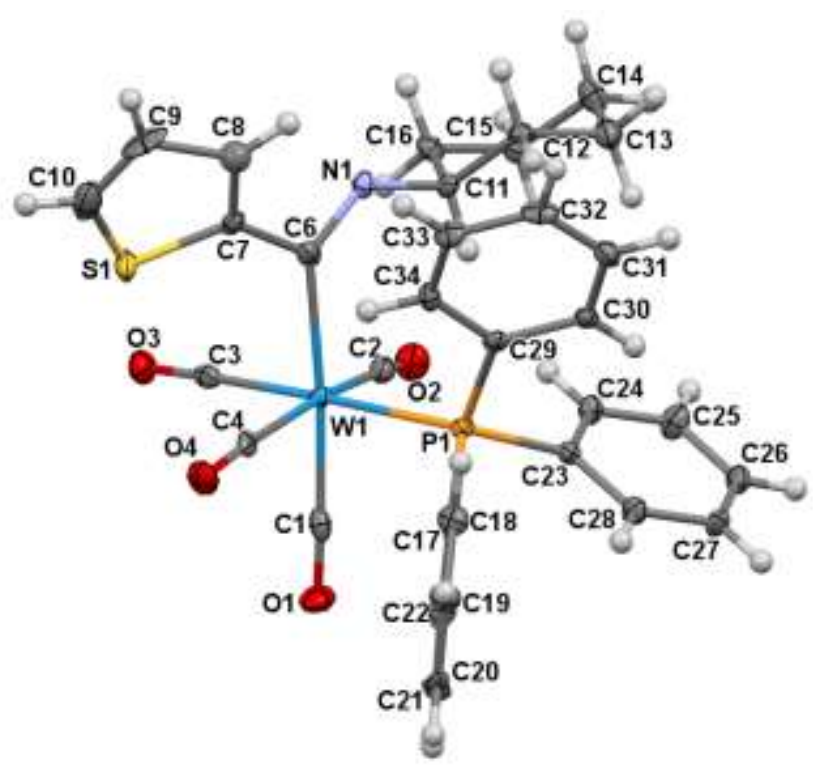

Figure 2. Perspective view of the major orientation of $\mathbf{3}$ with thermal ellipsoids drawn at the 50\% probability level 


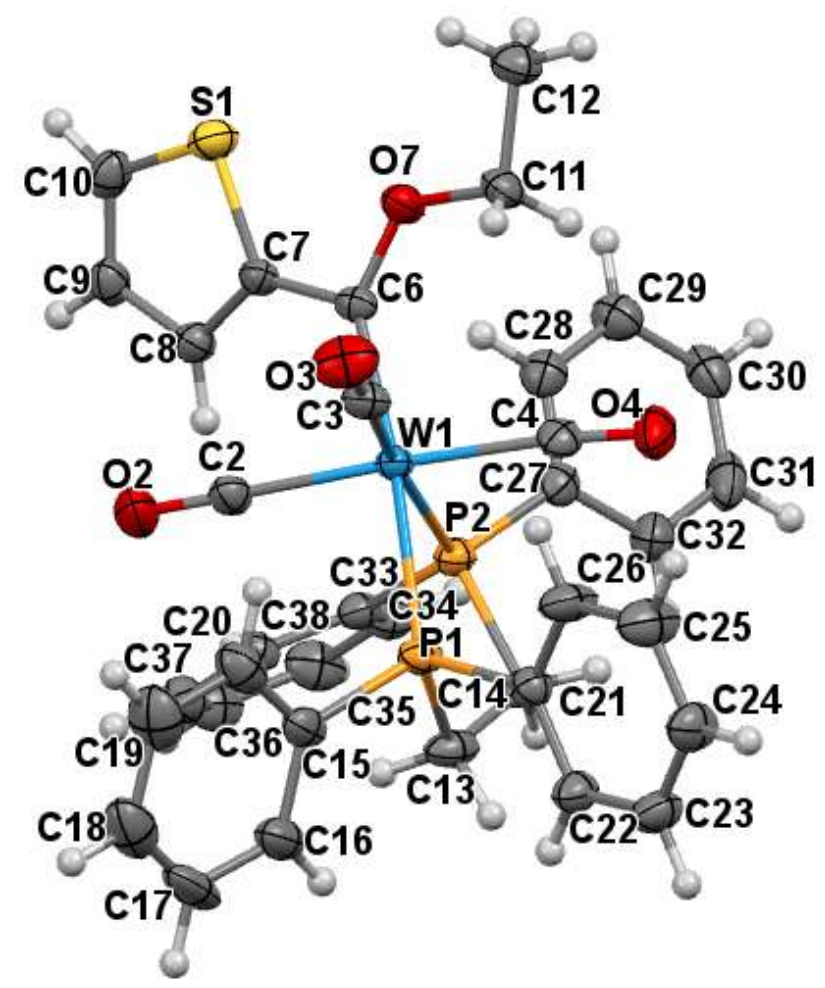

Figure 3. Perspective view of $\mathbf{5}$ with thermal ellipsoids drawn at the $50 \%$ probability level

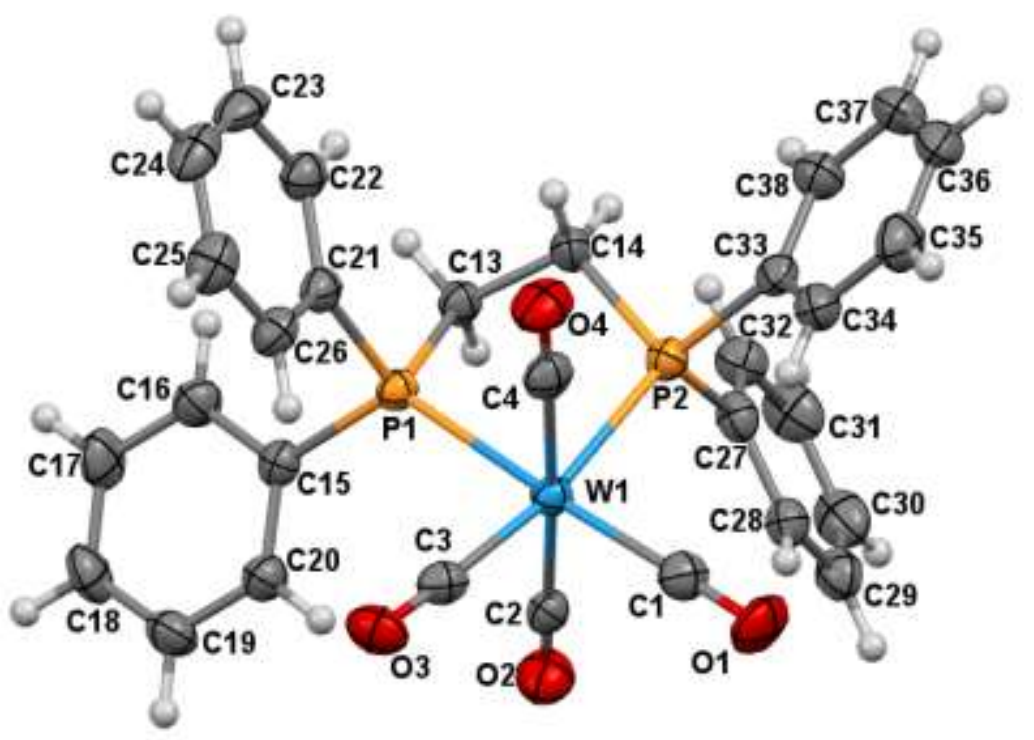

Figure 4. Perspective view of $\mathbf{E}$ with thermal ellipsoids drawn at the 50\% probability level

\subsection{CV and DFT calculations}

The cyclic voltammograms of carbene complexes 1-12 of this study are shown in Figure 5 and the data summarized in Table 1. Electrochemical data of complexes AD, $\left[\mathrm{W}(\mathrm{CO})_{5}\{\mathrm{C}(\mathrm{EDA}) \mathrm{Ar}\}\right](\mathrm{Ar}=\mathrm{Th}(\mathbf{F})$ or $\mathrm{Fu}(\mathbf{G}), \mathrm{EDA}=$ ethylene diamine $)$, as well as chelated products $\left[\mathrm{W}(\mathrm{CO})_{4}\{\mathrm{C}(\mathrm{EDA}) \mathrm{Ar}\}\right](\mathrm{Ar}=\mathrm{Th}(\mathbf{H})$ or $\mathrm{Fu}(\mathbf{I}))$, obtained under that same experimental conditions than complexes 1-12 [4], is also added for 
comparative purposes. One (5-12) or two (1-4) oxidation processes and one reduction (1-12) process are observed in the potential window of the solvent $\mathrm{CH}_{3} \mathrm{CN}$ used, indicated with (a), (b) and (c) in Figure 5. The linear sweep wave voltammetry (LSV) and peak current ratios ( $i_{\mathrm{pa}}$ of the oxidation process $/ i_{\mathrm{pc}}$ of the reduction process) of complexes 1-4 indicated a two-electron transfer for both oxidation processes relative to a one electron reduction process, see the peak current ratios in Table 1 and the LSV of complexes $\mathbf{1}$ and $\mathbf{4}$ in Figure 5 as examples. The LSV and peak current ratios of complexes 5-12, are consistent with a two-electron oxidation process and a oneelectron reduction process (Table 1 and LSV of 12trans in Figure 5). The peak current and LSV ratios are in some cases less than 2, but still larger than 1, possibly due to the irreversible nature of the oxidation process or decomposition of unstable charged species on the LSV time scale. The first two-electron oxidation process is in agreement with results of Licandro et. al. [60], who also suggested a two-electron metal-based oxidation process for the first oxidation, observed for a series of five alkoxy-, amino- and hydrazinocarbene complexes of tungsten. The two-electron stoichiometry of the irreversible $\mathrm{W}(0)$ carbene oxidation is in contrast with the reversible one-electron oxidation observed for pentacarbonyl $\mathrm{Cr}(0)$ carbenes $[1,3,60]$.

\subsubsection{Electrochemical oxidation}

The first oxidation process observed is assigned to the two-electron oxidation of the $\mathrm{W}(0)$ centre to $\mathrm{W}(\mathrm{II})$, indicated with peak (a) in Figure 5. The assignment that the oxidation is metal-based is supported by DFT calculations of this study, and in agreement with the experimental assignment of the oxidation of the series of carbene complexes of tungsten by Licandro et. al. [60] and two aminocarbene complexes of tungsten reported by Ludvík [61]. The second oxidation process observed involves the two-electron oxidation of the electrochemically generated W(II) centre to W(IV), indicated with peak (c) in Figure 5. This assignment is supported by DFT calculations of this study and has not previously been reported for tungsten-carbene complexes.

Oxidation of $\mathrm{W}(0)$ to $\mathrm{W}(\mathrm{II})$ of the monosubstituted phosphine- and phosphitecontaining carbenes (complexes 1-4 and 9-12) is observed at potentials between 0.229 and $0.420 \mathrm{~V} v s . \mathrm{Fc} / \mathrm{Fc}^{+}$and the $\mathrm{W}(\mathrm{II})$ to (IV) oxidation of 1-4 at potentials larger than 
$0.795 \mathrm{~V} v s . \mathrm{Fc} / \mathrm{Fc}^{+}$. Both $\mathrm{W}^{0 / I I}$ and $\mathrm{W}^{\mathrm{II} / \mathrm{IV}}$ oxidation processes are electrochemically and chemically irreversible. Electrochemical and chemical reversible redox processes are characterized by $\Delta E=E_{\mathrm{pa}}-E_{\mathrm{pc}}=0.059 \mathrm{~V}$ and $i_{\mathrm{pc}} i_{\mathrm{pa}}=1$ respectively [18-20]. $\mathrm{W}(0)$ to $\mathrm{W}(\mathrm{II})$ oxidation of the dppe-containing complexes 5-8 is observed at lower, more negative potentials than 1-4 and 9-12, at potentials between -0.152 and $-0.033 \mathrm{~V}$ vs. $\mathrm{FcH} / \mathrm{FcH}^{+}$.

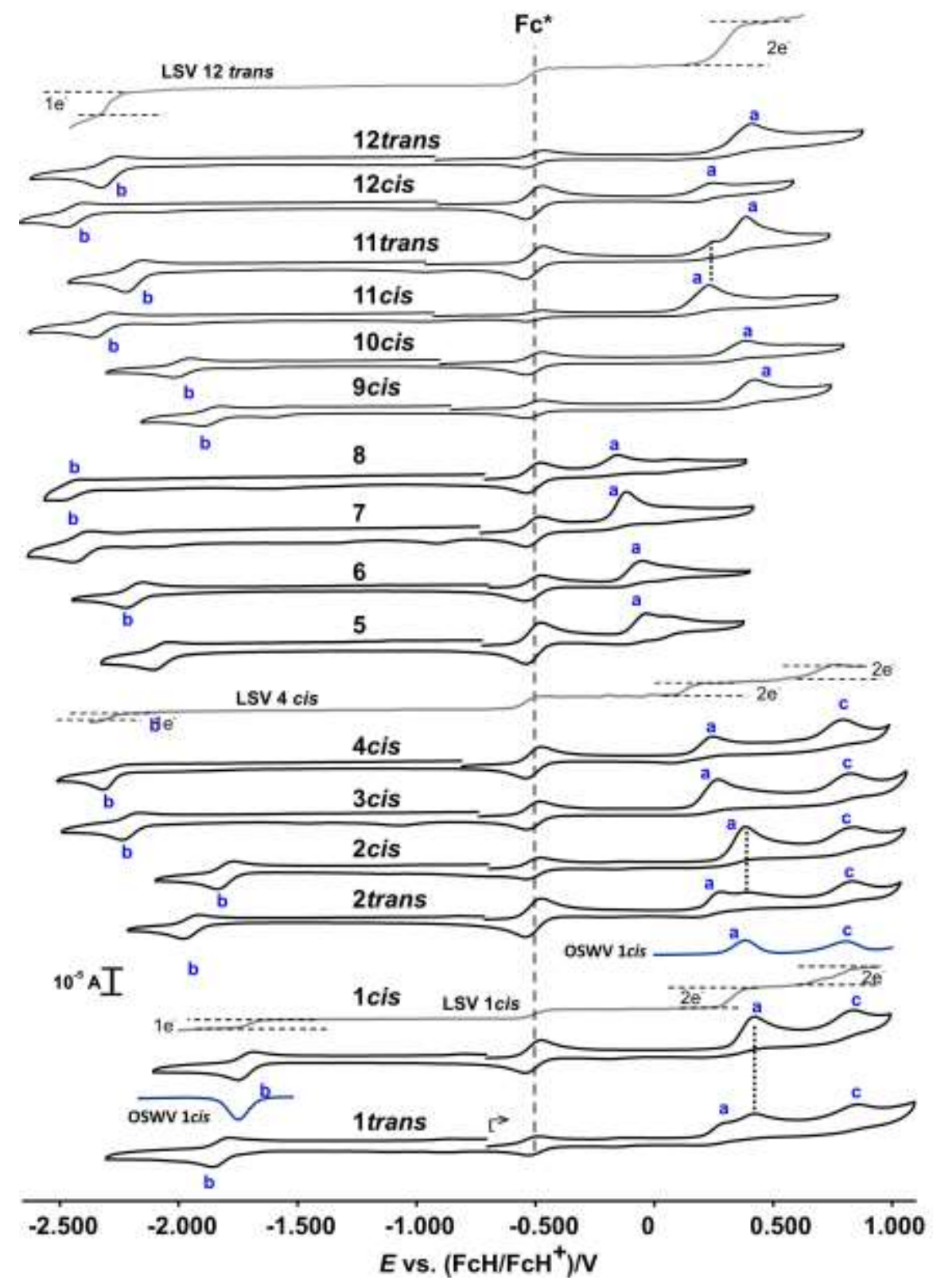

Figure 5. Cyclic voltammograms of c.a. $0.0005 \mathrm{~mol} \mathrm{dm}^{-3}$ solutions of 1-12 in $\mathrm{CH}_{3} \mathrm{CN} / 0.1 \mathrm{~mol} \mathrm{dm}^{-3}$ $\left[{ }^{\mathrm{n}}\left(\mathrm{Bu}_{4}\right) \mathrm{N}\right]\left[\mathrm{PF}_{6}\right]$ on a glassy carbon-working electrode at a scan rate of $0.100 \mathrm{~V} \mathrm{~s}^{-1} . \mathrm{CV}$ peaks due to the internal standard used, are marked $\mathrm{Fc}^{*}$. Scans initiated in the positive direction from c.a. $-0.700 \mathrm{~V}$. Oxidation peaks are marked with (a) and (c) and reduction peaks with (b). LSV's are shown for 1cis, 4 cis and 12trans. 


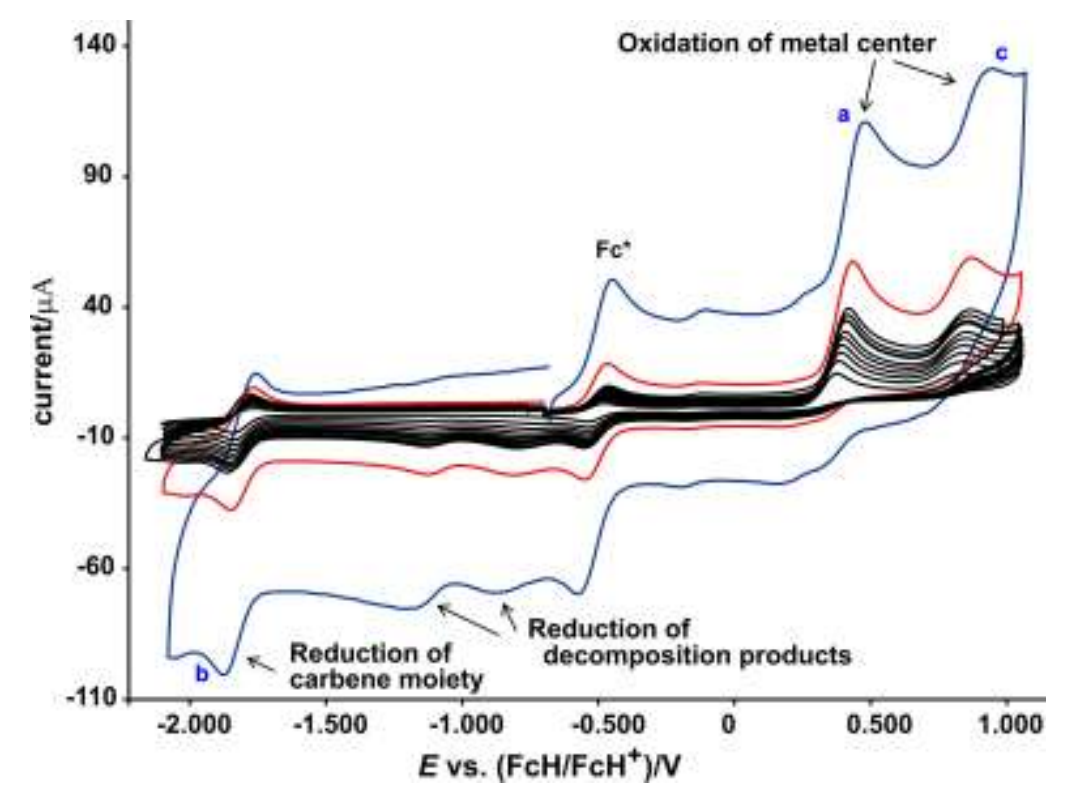

Figure 6. Cyclic voltammograms of c.a. $0.0005 \mathrm{~mol} \mathrm{dm}^{-3}$ solutions of 2 cis in $\mathrm{CH}_{3} \mathrm{CN} / 0.1 \mathrm{~mol} \mathrm{dm}^{-3}$ $\left[{ }^{\mathrm{n}}\left(\mathrm{Bu}_{4}\right) \mathrm{N}\right]\left[\mathrm{PF}_{6}\right]$ on a glassy carbon-working electrode at a scan rate of at scan rates of 0.050 (smallest currents) till $0.450 \mathrm{~V} \mathrm{~s}^{-1}$ in $0.050 \mathrm{~V}$ increments, $1.000 \mathrm{~V} \mathrm{~s}^{-1}$ (indicated in red) and $5.000 \mathrm{~V} \mathrm{~s}^{-1}$ (indicated in blue). Decamethylferrocene, $\mathrm{Fc}^{*}$, was used as internal standard. The small reduction peaks are ascribed to decomposition products that are generated during $\mathrm{W}$ oxidation since these peaks are absent if the scans are initiated in the negative direction. Scans initiated in the positive direction from $-0.700 \mathrm{~V}$.

\subsubsection{Electrochemical reduction}

The one-electron reduction processes observed at potentials below $-1.680 \mathrm{~V} v s$. $\mathrm{FcH} / \mathrm{FcH}^{+}$is labelled (b) in Figure 5. This process is ascribed to the reduction of the carbene ligand. This assignment is supported by DFT calculations of this study, and in agreement with the experimental assignment of the reduction centre of two aminocarbene complexes of tungsten reported by Ludvík [61]. These reduction processes, except for $\mathbf{4}$ cis, can be considered electrochemically reversible, since $\Delta E<$ 0.080 V for the phosphine-substituted carbene complexes 1-3 and 5-12 of this study. (Experimentally the peak separation is often larger than the Nernstian value of 0.059 $\mathrm{V}$ (for a one electron process) due to uncompensated ohmic drops in the cell $[62,63]$, therefore, for this study, $\Delta E_{\mathrm{p}}$ values up to $0.090 \mathrm{~V}$ will be considered as indicative of an electrochemically reversible couple.) The peak current ratio of the reduction process $i_{\mathrm{pa}(\mathrm{red})} / i_{\mathrm{pc}(\mathrm{red})}<<1$ but increases with an increase in scan rate, indicating that the reduced carbene radicals are stabilized long enough on the time scale of the $\mathrm{CV}$ to be re-oxidized, see Figure 6 for complex 2cis as an example. The reduction process 
can thus be considered as chemically quasi reversible for carbene complexes 1-3 and 5-12 of this study.

Table 1. Cyclic voltammetry data of c.a. $0.0005 \mathrm{~mol} \mathrm{dm}^{-3}$ solutions of 1-12, A-D and F-I in $\mathrm{CH}_{3} \mathrm{CN}$ containing $0.1 \mathrm{~mol} \mathrm{dm}^{-3}\left[\mathrm{~N}\left({ }^{n} \mathrm{Bu}\right)_{4}\right]\left[\mathrm{PF}_{6}\right]$ as supporting electrolyte at a scan rate of $0.100 \mathrm{~V} \mathrm{~s}^{-1}$ and 20 ${ }^{\circ} \mathrm{C}$. Potentials are reported in $\mathrm{V}$ relative to the $\mathrm{FcH} / \mathrm{FcH}^{+}$couple.

\begin{tabular}{|c|c|c|c|c|c|c|c|c|c|}
\hline & \multicolumn{3}{|c|}{ Oxidation } & \multicolumn{3}{|c|}{ Reduction } & \multirow[b]{2}{*}{$E^{0^{\prime}} / \mathrm{V}$} & \multirow[b]{2}{*}{$i_{\mathrm{pc}} / \mu \mathrm{A}$} & \multirow[b]{2}{*}{$i_{\mathrm{pal}(\mathrm{ox})} / i_{\mathrm{pa}(\mathrm{red})}$} \\
\hline & $E_{\mathrm{pa} 1} / \mathrm{V}$ & $E_{\mathrm{pa} 2} / \mathrm{V}$ & $i_{\mathrm{pa} 1} / \mu \mathrm{A}$ & $E_{\mathrm{pa}} / \mathrm{V}$ & $E_{\mathrm{pc}} / \mathrm{V}$ & $\Delta E / \mathrm{V}$ & & & \\
\hline 1trans & 0.315 & 0.857 & $7.6^{\mathrm{a}}$ & -1.785 & -1.857 & 0.072 & -1.821 & 4.8 & - \\
\hline 1cis & 0.421 & 0.839 & 8.6 & -1.683 & -1.751 & 0.068 & -1.717 & 4.5 & 1.9 \\
\hline 2 trans & 0.281 & 0.835 & $9.3^{\mathrm{a}}$ & -1.915 & -1.981 & 0.066 & -1.948 & 6.5 & - \\
\hline $2 c i s$ & 0.383 & 0.839 & 16.9 & -1.767 & -1.837 & 0.070 & -1.802 & 8.3 & 2.0 \\
\hline 3cis & 0.268 & 0.826 & 9.4 & -2.160 & -2.238 & 0.078 & -2.199 & 4.6 & 2.0 \\
\hline $4 c i s$ & 0.245 & 0.795 & 6.1 & - & -2.317 & - & - & 4.6 & 1.3 \\
\hline 5 & -0.033 & - & 5.3 & -2.038 & -2.110 & 0.072 & -2.074 & 3.4 & 1.6 \\
\hline 6 & -0.049 & - & 10.0 & -2.151 & -2.223 & 0.072 & -2.187 & 5.6 & 1.8 \\
\hline 7 & -0.116 & - & 7.1 & -2.370 & -2.444 & 0.074 & -2.407 & 3.7 & 1.9 \\
\hline 8 & -0.152 & - & 3.5 & -2.420 & -2.530 & 0.054 & -2.447 & $2.3^{\mathrm{c}}$ & - \\
\hline 9cis & 0.419 & - & 8.3 & -1.827 & -1.903 & 0.076 & -1.865 & 3.4 & 2.4 \\
\hline 10cis & 0.384 & - & 5.8 & -1.954 & -2.022 & 0.068 & -1.988 & 3.1 & 1.9 \\
\hline 11trans & 0.384 & - & $11.0^{\mathrm{a}}$ & -2.154 & -2.226 & 0.072 & -2.190 & 7.0 & - \\
\hline 11cis & 0.229 & - & 10.1 & -2.289 & -2.363 & 0.074 & -2.326 & 5.0 & 2.0 \\
\hline 12trans & 0.408 & - & $11.2^{\mathrm{a}}$ & -2.250 & -2.330 & 0.080 & -2.291 & 7.3 & - \\
\hline 12cis & 0.248 & - & 5.2 & -2.398 & -2.478 & 0.080 & -2.438 & $4.9^{\mathrm{c}}$ & - \\
\hline$A^{c}$ & 0.728 & - & 18.9 & -1.478 & -1.564 & 0.086 & -1.521 & 10.0 & 1.9 \\
\hline $\mathbf{B}^{\mathbf{c}}$ & 0.697 & - & 24.8 & -1.559 & -1.645 & 0.086 & -1.602 & 14.1 & 1.8 \\
\hline $\mathbf{C}^{\mathbf{c}}$ & 0.562 & - & 15.7 & -2.028 & -2.098 & 0.070 & -2.063 & 7.8 & 2.0 \\
\hline $\mathbf{D}^{\mathbf{c}}$ & 0.620 & - & 10.9 & -2.134 & -2.200 & 0.066 & -2.167 & 6.6 & 1.6 \\
\hline $\mathbf{F}^{\mathbf{c}}$ & 0.404 & - & 5.5 & - & -2.080 & - & - & 4.0 & 1.4 \\
\hline $\mathbf{G}^{\mathbf{c}}$ & 0.498 & - & 10.4 & - & -2.174 & - & - & 12.6 & 0.8 \\
\hline $\mathbf{H}^{\mathbf{c}}$ & 0.005 & - & 9.0 & - & -2.203 & - & - & 11.3 & 0.8 \\
\hline $\mathbf{I}^{\mathbf{a}}$ & 0.020 & - & 6.4 & - & -2.330 & - & - & 5.7 & 1.1 \\
\hline
\end{tabular}

\subsubsection{DFT calculations}

To understand the oxidation and reduction processes observed a density functional theory study of complexes $\mathbf{1 - 1 2}$ is presented. W(0)-carbenes are low spin diamagnetic 
$\mathrm{d}^{6}$ complexes. In an octahedral ligand field, the expected d-orbital occupation for 1-12 is $t_{2 \mathrm{~g}}{ }^{6} e_{\mathrm{g}}{ }^{0}$ or $d_{x y}{ }^{2} d_{x z}{ }^{2} d_{y z}{ }^{2} d_{x 2-y 2}{ }^{0} d_{z 2}{ }^{0}$. To illustrate the role of the HOMO and the LUMO in the oxidation and reduction processes, the frontier molecular orbitals (MOs) of complexes 1cis, 1trans and 5mer are shown in Figure 7 as representative examples of the frontier MOs of 1-12. The HOMO, HOMO-1 and HOMO-2 are all mainly W-dorbital based, while the frontier LUMOs are ligand based. The LUMO is distributed mainly over the carbene carbon, the nitrogen or oxygen heteroatom and the furyl or thienyl substituent. The LUMO+1 is located on the $\mathrm{CO}$ and phenyl groups, while the LUMO+2 is located mainly on the phenyl groups attached to the phosphorus atom.

The oxidation of a complex involves the removal of an electron from the highest occupied orbital (HOMO) of the complex, leading to a positively charged cation. The character of the HOMO will thus show where the oxidation process will take place and the energy of the HOMO will be related to the ease of oxidation. From Figure 8 it is clear that the HOMOs of all the complexes 1-12 of this study is mainly located on the W-metal ion, with a small amount of $\mathrm{d}_{\pi}-\mathrm{p}_{\pi}$ bonding to one carbonyl carbon atom. Thus oxidation of carbenes 1-12 will involve the W-metal atom, with the formation of either a diamagnetic W(II) (closed shell singlet state, $d_{x y}{ }^{2} d_{x z}{ }^{2} d_{y z}{ }^{0} d_{x 2-y 2}{ }^{0} d_{z 2}{ }^{0}$ or an open shell singlet $d_{x y}{ }^{2} d_{x z}{ }^{1} d_{y z}{ }^{1} d_{x 2-y 2}{ }^{0} d_{z 2}{ }^{0}$ ) or a paramagnetic W(II) (triplet state, $d_{x y}{ }^{2} d_{x z}{ }^{1} d_{y z}{ }^{1} d_{x 2 \text { - }}$ ${ }_{y 2}{ }^{0} d_{z 2}{ }^{0}$ ) cation with charge +2 . DFT calculations showed that the W(II) closed shell singlet state is more stable than the open shell singlet or the triplet state by more than $0.4 \mathrm{eV}$. The illustration of the metal-d based MOs of the optimized geometry of the diamagnetic $\mathrm{W}(\mathrm{II})$ (singlet state, $\mathrm{S}=0$ ) for complex 1trans as an representative example, in Figure 9, confirms the occupation $d_{x y}{ }^{2} d_{x z}{ }^{2} d_{y z}{ }^{0} d_{x 2-y 2}{ }^{0} d_{z 2}{ }^{0}$. 


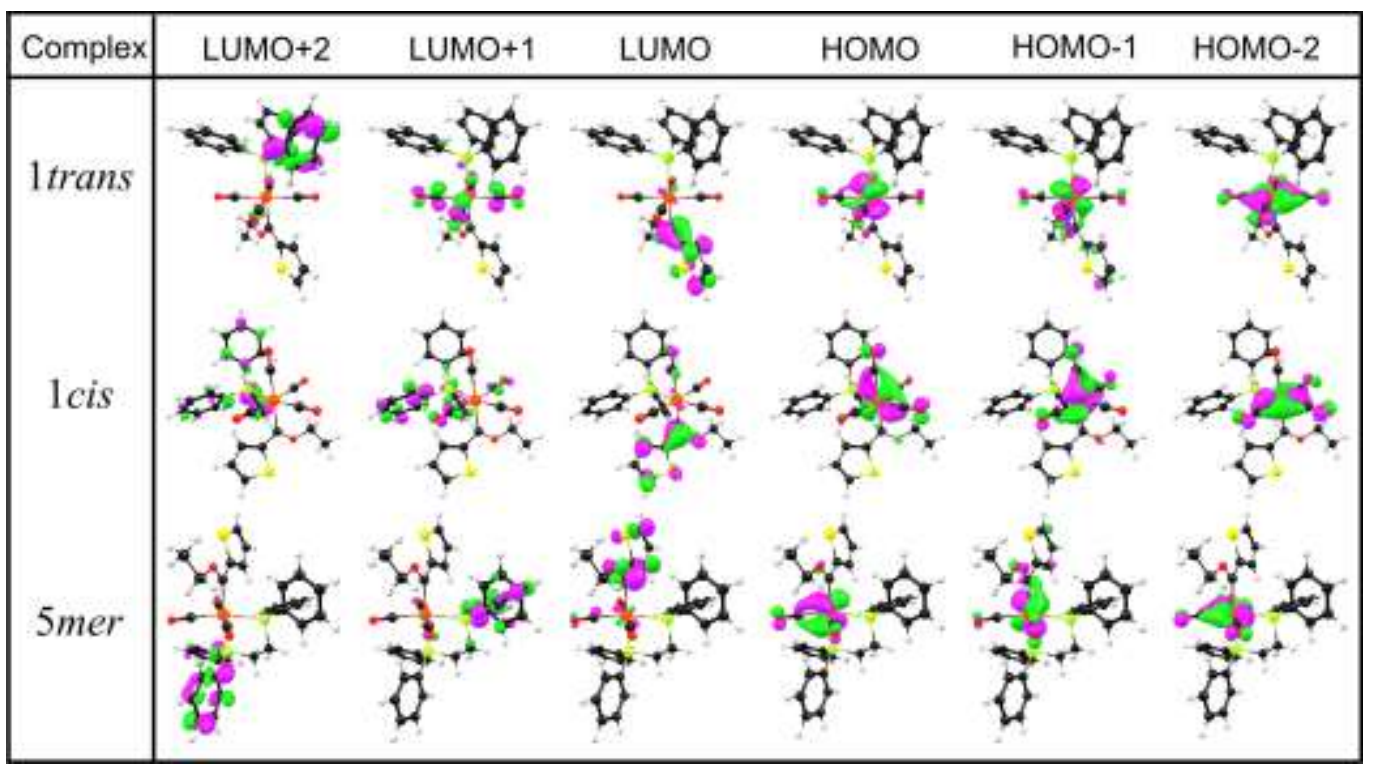

Figure 7. Visualization of frontier MOs of the neutral complex 1trans, 1cis and 5mer. The MO plots use a contour of $60 \mathrm{e} / \mathrm{nm}^{3}$.

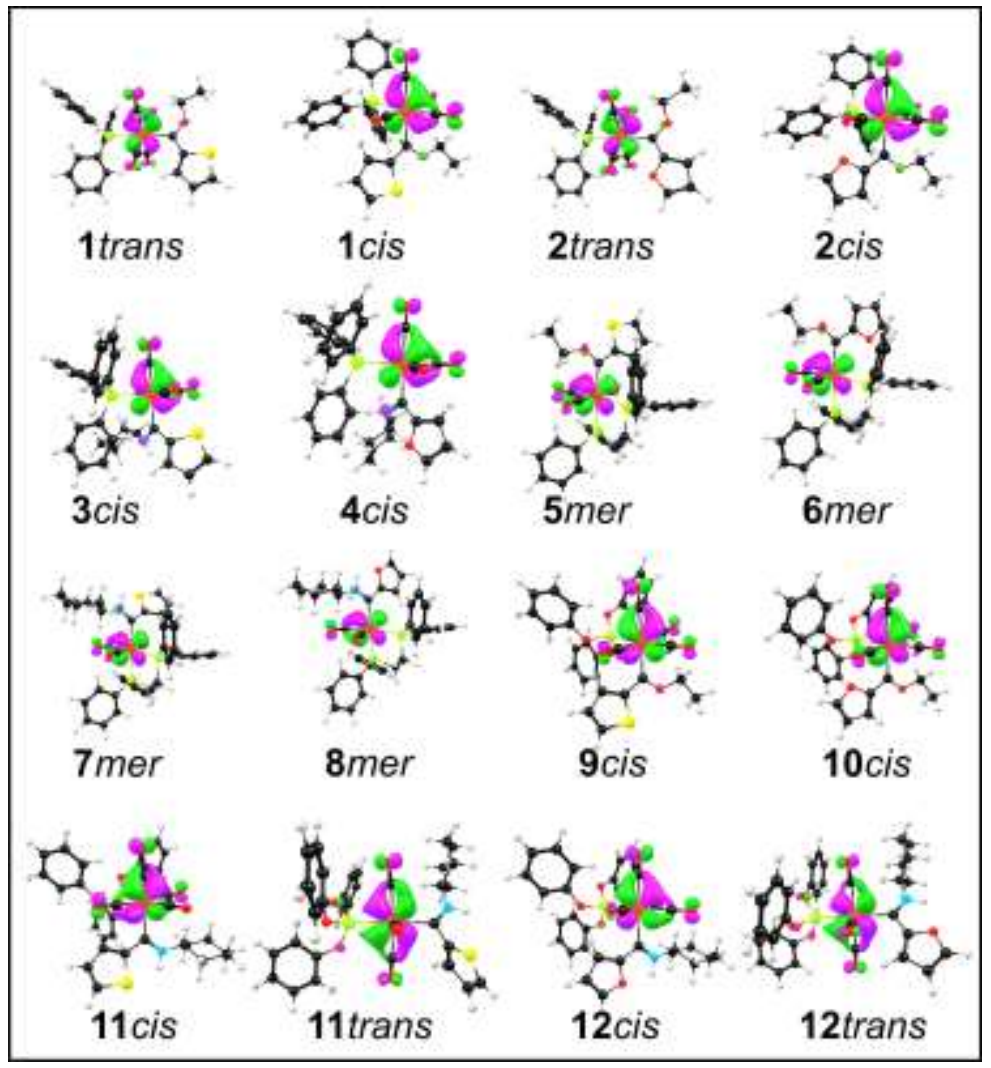

Figure 8. Visualization of the HOMOS of the neutral complexes of 1-12. The MO plots use a contour of $60 \mathrm{e} / \mathrm{nm}^{3}$. 


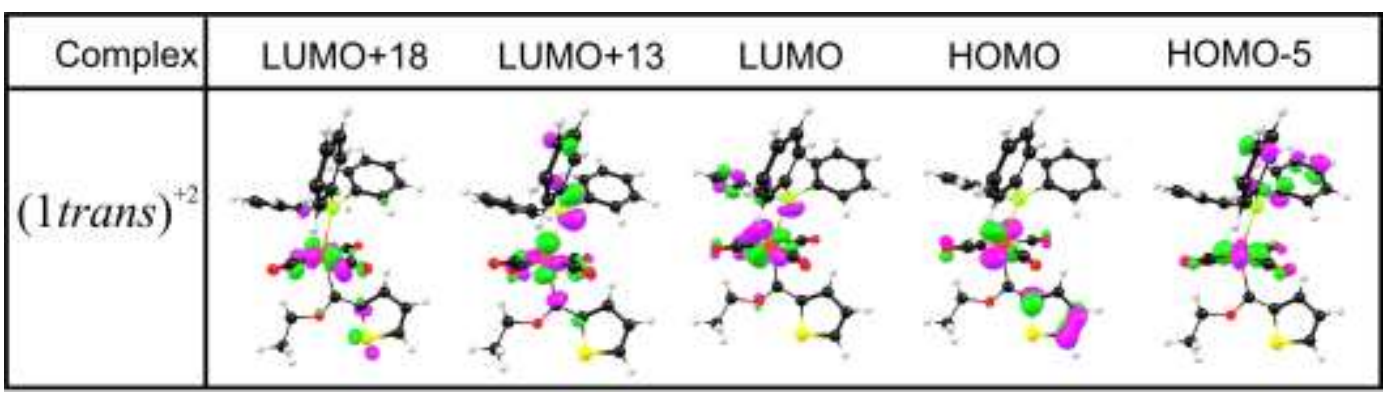

Figure 9. Visualization of the d-based MOs of the doubly oxidized complex 1trans. The MO plots use a contour of $60 \mathrm{e} / \mathrm{nm}^{3}$.

Since reduction of a complex involves the addition of an electron to the LUMO of the complex, the character of the LUMO shows where the reduction process will take place and the energy of the LUMO is related to the ease of reduction. Figure 7 shows clearly that the $\mathrm{W}$-metal is not involved in the reduction at all; all three frontier LUMOs are ligand-based. The LUMO itself mainly involves the carbene carbon, the nitrogen or oxygen heteroatom and the furyl or thienyl substituent, implying that the electron donating properties of $\mathrm{N} v s$. $\mathrm{O}$ and furyl $v s$. thienyl will influence the ease of reduction. The localization of the added unpaired electron of the reduced anion can be visualized by the HOMO of the reduced species or by a spin-density plot of the reduced species (charge $q=-1$, spin $S=1 / 2$ ), see Figure 10 for complexes 2, 6 and 9 as representative examples. From Figure 10 is it clear that the reduction centre is distributed mainly over the carbene carbon (the Mulliken spin density on the carbene carbon of the reduced complex is between 0.36 and 0.54 electrons, see Table 2), as well as on the nitrogen or oxygen heteroatom and the furyl or thienyl substituent. 


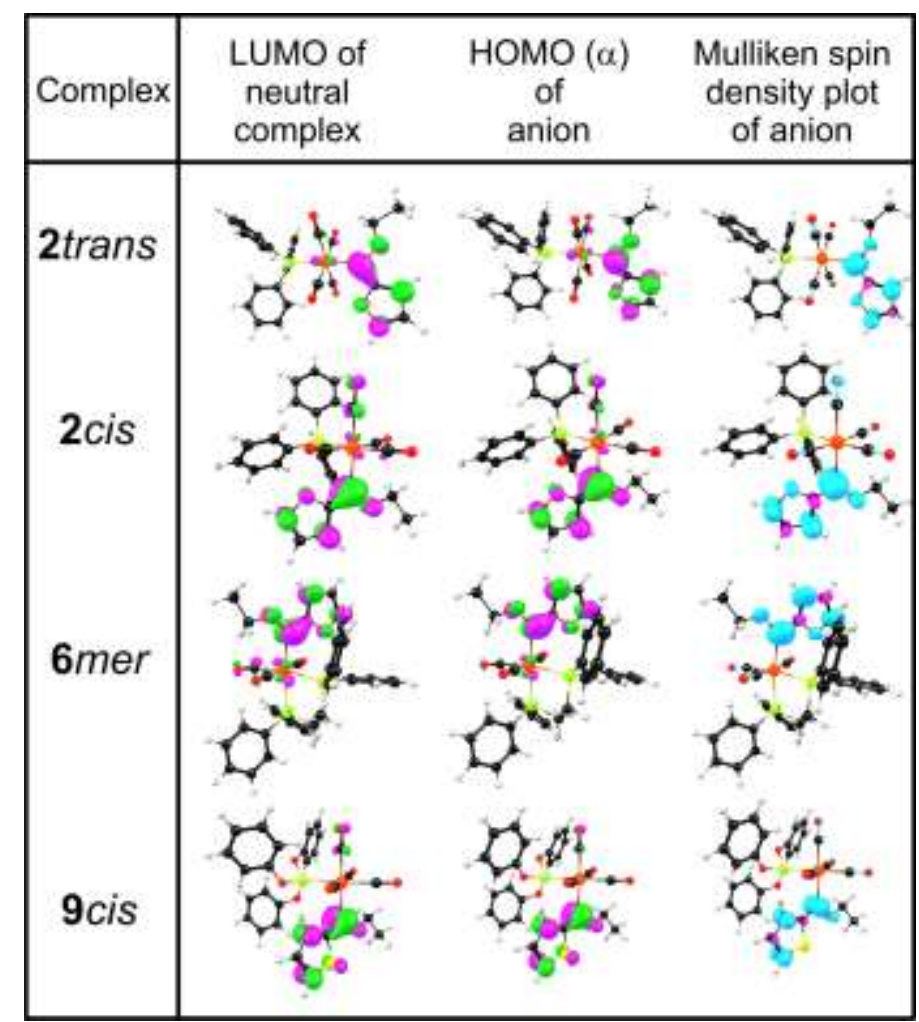

Figure 10. Visualization of (a) the LUMO of the neutral complexes of $\mathbf{2 ,} 6$ and $\mathbf{9}$ and (b) the HOMO and (c) spin density plot of the reduced radical anion $(q=-1, S=1 / 2)$ of complexes of $\mathbf{2}, \mathbf{6}$ and $\mathbf{9}$. The MO and spin density plots use a contour of 60 and $6 \mathrm{e} / \mathrm{nm}^{3}$ respectively.

\subsubsection{Substituent's effect on oxidation and reduction potential}

After having confirmed the nature of the redox processes observed in Figure 5 using a DFT study, a few remarks relating to the substituent's effect observed for the oxidation and reduction of phosphine-substituted W-carbene complexes follow.

On comparing $\mathrm{PPh}_{3}$-containing complexes 1-4 $\left(\left[(\mathrm{CO})_{4}\left(\mathrm{PPh}_{3}\right) \mathrm{W}=\mathrm{C}(\mathrm{X}) \mathrm{R}\right]\right)$ with dppe-containing complexes 5-8 $\left(\left[(\mathrm{CO})_{3}(\mathrm{dppe}) \mathrm{W}=\mathrm{C}(\mathrm{X}) \mathrm{R}\right]\right)$, it is observed that the dppe-containing complexes are considerably easier to oxidize than the $\mathrm{PPh}_{3}$ containing complexes, with oxidation potentials of more than $0.275 \mathrm{~V}$ lower than that of the $\mathrm{PPh}_{3}$-containing complexes. The oxidation potential of the $\mathrm{P}(\mathrm{OPh})_{3}$-containing complexes 9-12 $\left(\left[(\mathrm{CO})_{4}\left(\mathrm{P}(\mathrm{OPh})_{3}\right) \mathrm{W}=\mathrm{C}(\mathrm{X}) \mathrm{R}\right]\right)$ lies in the same range as the oxidation potential of complexes 1-4. Since the oxidation centre is mainly located on the Wmetal centre, it is expected that the electronic influence of any group or ligand directly attached to the $\mathrm{W}$ atom will be more pronounced than the influence of substituents on the carbene ligand. $\mathrm{PR}_{3}^{\prime}$ is a weaker $\pi$-acceptor ligand than $\mathrm{CO}$. The $(\mathrm{CO})_{4}\left(\mathrm{PR}_{3}\right)\left(\mathrm{R}^{\prime}=\right.$ 
$\mathrm{Ph}$ or $\mathrm{OPh}$ ) ligand combination will thus withdraw more electron density from the Wmetal centre relative to the $(\mathrm{CO})_{3}(\mathrm{dppe})$ ligand combination with two phosphorus atoms coordinated to $\mathrm{W}$. With relatively less electron density on $\mathrm{W}$, oxidation of 1-4 and 9-12 is thus more difficult, at a higher potential. The same trend is observed for the reduction potential, although less pronounced. This is expected, since the reduction is not metal-based. The W-metal in the pentacarbonyl complexes A-D and F-I with no phosphorus atom coordinated to $\mathrm{W}$, has the highest oxidation potential. The effect of $\mathrm{CO}$ substitution in pentacarbonyl Fischer carbene complexes on the ease of oxidation, reveals the following trend for the sixteen complexes in Table 1:

- Largest $E_{\mathrm{pa}}$, most difficult to oxidize: $\left[(\mathrm{CO})_{5} \mathrm{~W}\right.$-carbene $]>\left[(\mathrm{CO})_{4}\left(\mathrm{PR}_{3}{ }_{3}\right) \mathrm{W}\right.$ carbene $]>\left[(\mathrm{CO})_{4}(\mathrm{EDA}) \mathrm{W}\right.$-carbene $]>\left[(\mathrm{CO})_{3}(\mathrm{dppe}) \mathrm{W}\right.$-carbene $]$ (smallest $E_{\mathrm{pa}}$, easiest to oxidize).

This order is the similar to the order of the $\pi$-acceptor ability of the ligands attached to $\mathrm{W}$, when comparing the $v_{\mathrm{CO}} \mathrm{IR}$ stretching frequency of the $\mathrm{A}_{1(\mathrm{eq})}$ bands of the respective complexes:

- Highest $v_{\mathrm{CO}}$, best $\pi$-acceptor: $\left[(\mathrm{CO})_{5} \mathrm{~W}\right.$-carbene $]>\left[(\mathrm{CO})_{4}\left(\mathrm{PR}_{3}^{\prime}\right) \mathrm{W}\right.$-carbene $] \approx$ $\left[(\mathrm{CO})_{4}(\mathrm{EDA}) \mathrm{W}\right.$-carbene $]>\left[(\mathrm{CO})_{3}(\mathrm{dppe}) \mathrm{W}\right.$-carbene $]\left(\right.$ lowest $\left.v_{\mathrm{CO}}\right)$.

DFT results on the relative energies of the $f a c$ and mer isomers of 5-8, indicated that the mer isomers are considerably more stable than the $f a c$ isomers. The calculated Boltzmann population of the fac isomers are less than $10 \%$. Experimentally the mer isomer is the main isomer observed. The decay process of the $\mathrm{W}(0)-\mathrm{W}(\mathrm{II})$ oxidation of some of the mer isomers of $\mathbf{5 - 8}$ showed a small shoulder that is consistent with a small amount of $f a c$ isomer being oxidized at a higher potential. A calculated higher electronic energy and lower HOMO energy of the fac isomer, relative to that of the mer isomer, is consistent with this observation.

In comparing the thienyl $v s$. furyl substituent (compare 1 to 2,3 to 4,5 to 6,7 to 8,9 to $\mathbf{1 0}$ and $\mathbf{1 1}$ to $\mathbf{1 2}$ ), we observe that the oxidation potential is very similar for the thienyl vs. furyl complexes (within $0.04 \mathrm{~V}$ ). However, the reduction peak of the 
reduction process shifts more than $0.080 \mathrm{~V}$ to a lower potential in changing from thienyl to furyl. The furyl-containing complexes are thus reduced at a lower, more negative $E_{\mathrm{pc}}$. Since the reduction centre is distributed over the carbene carbon, the nitrogen or oxygen heteroatom and the furyl or thienyl substituent, the electronic properties of the thienyl or furyl substituent will directly influence the reduction potential to a larger extent than the oxidation potential which is $\mathrm{W}$-metal based. The effect of the thienyl substituent compared to furyl, can be calculated by the electronic population of the carbene carbon (Mulliken charge and NPA charge higher for thienyl) and experimentally seen by the ${ }^{13} \mathrm{C}$ shift of the carbene carbon (5-26 ppm to lower field for thienyl), see Table 2.

In comparing the alkoxy- vs. aminocarbene (compare 1 to 3,2 to 4,5 to 7,6 to 8 , 9cis to 11cis and 10cis to 12cis), we observe that the alkoxycarbenes are more difficult to oxidize (higher, more positive $E_{\mathrm{pa}}$ ) and more easily reduced (higher, less negative $\left.E_{\mathrm{pc}}\right)$ than the aminocarbenes. This is consistent with the higher electronegativity of the oxygen atom (3.5) with respect to nitrogen (3.1) [64], making the carbene carbon less negative and more difficult to oxidize (easier to reduce). The effect on the reduction potential is more pronounced since the reduction centre is spread over the carbene carbon, the nitrogen or oxygen heteroatom and the furyl or thienyl substituent. The influence of the electron withdrawing power of $\mathrm{O}$ compared to $\mathrm{N}$ on the carbene carbon in the neutral complex is demonstrated by calculating the natural population analysis (NPA) charge on the carbene carbon, which is much lower for the aminocarbenes $(0.14-0.22 \mathrm{au})$ than for the alkoxycarbenes $(0.31-0.39 \mathrm{au})$, see the data in Table 2 . In the reduced complex, the NPA charge on the carbene carbon reduces further to $-0.06-0.06$ au for the aminocarbenes and $0.11-0.20$ au for the alkoxycarbenes. The calculated Mulliken spin population on the carbene carbon of the reduced complex is also generally higher for the aminocarbenes than for the corresponding alkoxycarbenes, see the data in Table 2. Experimentally the ${ }^{13} \mathrm{C}$ shift of the carbene carbon follows the same trend; 223 - $249 \mathrm{ppm}$ for the aminocarbenes and 279 - $296 \mathrm{ppm}$ for the alkoxycarbenes of this study. Both experimental and calculated results presented above show that the influence of the heteroatom directly bonded to the carbene carbon ( $\mathrm{O}$ of alkoxy vs. $\mathrm{N}$ of amino) on the reduction potential 
is more pronounced than the influence of the thienyl vs. furyl substituent with the $\mathrm{S}$ or O two bonds away from the carbene carbon.

The trans $-\left[(\mathrm{CO})_{4}\left(\mathrm{PPh}_{3}\right) \mathrm{W}=\mathrm{C}(\mathrm{OEt}) \mathrm{R}\right]$ complexes 1trans and 2trans are easier to oxidize at a lower potential $(0.100 \mathrm{~V}$ lower) than the corresponding cis complexes. This is consistent with DFT calculations showing the trans complexes to be more than $0.1 \mathrm{eV}$ less stable than the corresponding cis complexes, and that the calculated HOMO energy of the trans complexes is $0.2 \mathrm{eV}$ higher than that of the corresponding cis complexes. Experimentally it is found that the trans complexes isomerize to the more stable cis complexes, explaining the shoulder on the oxidation peak on the CV of 1 cis and 2cis. In contrast, the oxidation potential of the trans$\left[(\mathrm{CO})_{4}\left(\mathrm{P}(\mathrm{OPh})_{3}\right) \mathrm{W}=\mathrm{C}(\mathrm{NHCy}) \mathrm{R}\right]$ complexes 11trans and 12trans is higher than the oxidation potential of the corresponding cis- $\left[(\mathrm{CO})_{4}\left(\mathrm{P}(\mathrm{OPh})_{3}\right) \mathrm{W}=\mathrm{C}(\mathrm{NHCy}) \mathrm{R}\right]$ complexes. The oxidation potential of the trans $-\left[(\mathrm{CO})_{4}\left(\mathrm{P}(\mathrm{OPh})_{3}\right) \mathrm{W}=\mathrm{C}(\mathrm{NHCy}) \mathrm{R}\right]$ complexes 11trans and 12trans is more than $0.06 \mathrm{~V}$ higher that the oxidation potential of the trans-[(CO) $\left.)_{4}\left(\mathrm{PPh}_{3}\right) \mathrm{W}=\mathrm{C}(\mathrm{OEt}) \mathrm{R}\right]$ complexes 1trans and 2trans. This result illustrates the effect of the electron-withdrawing nature of the $\mathrm{P}(\mathrm{OPh})_{3}$ group (better $\pi$-acceptor) over that of $\mathrm{PPh}_{3}$, making the $\mathrm{W}$-metal relatively more positive and more difficult to oxidize. This is in spite of the fact that the aminocarbenes are easier to oxidize than alkoxycarbenes. This result is in agreement with the oxidation potential of trans $-\left[\mathrm{Cr}(\mathrm{CO})_{4}\left(\mathrm{P}(\mathrm{OPh})_{3}\right)_{2}\right]$ being $0.46 \mathrm{~V}$ larger than that of trans$\left.\left[\mathrm{Cr}(\mathrm{CO})_{4}\left(\mathrm{PPh}_{3}\right)_{2}\right)\right][65]$. The calculated NPA charges on $\mathrm{W}$ for phosphine-carbene (14) and phosphite-carbene (9-12) complexes illustrate the better $\pi$-acceptor ability of $\mathrm{P}(\mathrm{OPh})_{3}$ compared to $\mathrm{PPh}_{3}$ :

- Lowest, most negative $\mathrm{q}_{\mathrm{NPA}}(\mathrm{W})$ : trans- $\left[(\mathrm{CO})_{4}\left(\mathrm{P}(\mathrm{OPh})_{3}\right) \mathrm{W}\right.$-carbene $]<$ trans$\left[(\mathrm{CO})_{4}\left(\mathrm{PPh}_{3}\right) \mathrm{W}\right.$-carbene $]$ (highest $\left.\mathrm{q}_{\mathrm{NPA}}(\mathrm{W})\right)$

- Lowest, most negative $\mathrm{q}_{\mathrm{NPA}}(\mathrm{W}):$ cis- $\left[(\mathrm{CO})_{4}\left(\mathrm{P}(\mathrm{OPh})_{3}\right) \mathrm{W}\right.$-carbene $]<$ cis$\left[(\mathrm{CO})_{4}\left(\mathrm{PPh}_{3}\right) \mathrm{W}\right.$-carbene $]$ (highest $\left.\mathrm{q}_{\mathrm{NPA}}(\mathrm{W})\right)$ 
Table 2. Experimental and density functional theory calculated characteristics of complexes of 1-12, A-D and F-I.

\begin{tabular}{|c|c|c|c|c|c|c|c|c|c|c|c|c|c|c|c|c|c|c|}
\hline $\mathbf{n r}$ & $\begin{array}{l}\text { Epa }(\text { ox }) \\
\text { / volt }\end{array}$ & $\begin{array}{l}\text { Epc } \\
\text { (red) / } \\
\text { volt }\end{array}$ & $\begin{array}{l}I P_{2 \mathrm{e}-} \\
\mathrm{eV}\end{array}$ & $\begin{array}{l}E A_{1 \mathrm{e}-} / \\
\mathrm{eV}\end{array}$ & $\begin{array}{l}E_{\text {Номо }} \\
/ \mathrm{eV}\end{array}$ & $\begin{array}{l}E_{\mathrm{LUMO}} \\
/ \mathrm{eV}\end{array}$ & $\mathbf{q}_{\text {Mull }}(\mathbf{W})$ & $\begin{array}{l}\mathbf{q}_{\mathrm{NPA}}(\mathbf{W}) \\
/ \mathrm{au}\end{array}$ & $\begin{array}{l}\mathbf{q}_{\text {Mull }} \\
\left(\mathbf{C}_{\text {carbene }}\right) \\
\text { / au }\end{array}$ & $\begin{array}{l}\mathbf{q}_{\mathrm{NPA}} \\
\left(\mathbf{C}_{\text {carbene }}\right) \\
/ \text { au }\end{array}$ & $\begin{array}{l}\mathbf{I R}^{a} \\
\left(\mathbf{c m}^{-1}\right) \\
\mathbf{A}_{1}^{(1)} \\
(\mathbf{e q})\end{array}$ & $\begin{array}{l}\text { IR } \\
\left(\mathbf{c m}^{-1}\right) \\
A_{1}(2) \\
(\mathbf{a x})\end{array}$ & $\begin{array}{l}{ }^{13} \mathrm{C}(\mathrm{ppm}) \\
\mathrm{CO}(\mathrm{eq}) \\
\text { trans to } \\
\mathrm{CO}\end{array}$ & $\begin{array}{l}{ }^{13} \mathrm{C} \\
\text { (ppm) } \\
\mathrm{CO}(\mathrm{eq}) \\
\text { trans to } \\
\mathrm{P} / \mathrm{N}\end{array}$ & $\begin{array}{l}{ }^{13} \mathrm{C} \\
(\mathbf{p p m}) \\
\mathrm{CO}(\mathbf{a x})\end{array}$ & $\begin{array}{l}{ }^{13} \mathrm{C} \quad(\mathbf{p p m}) \\
\mathrm{C}(\text { carbene })\end{array}$ & $\begin{array}{l}\mathbf{s p i n}_{\text {Mull }} \\
\left(\mathbf{C}_{\text {carbene, }}\right. \\
\text { reduced }) \\
/ \mathbf{e}\end{array}$ & $\begin{array}{l}\mathbf{q}_{\mathrm{NPA}} \\
\left(\mathbf{C}_{\text {carbene }}\right. \\
\text { reduced } \\
\text { au }\end{array}$ \\
\hline $01 c$ & 0.421 & -1.715 & 15.713 & 1.389 & -5.30 & -2.38 & -0.268 & -1.628 & 0.084 & 0.334 & 2009 & 1894 & 203.8 & 207.3 & 211.4 & 294.5 & 0.472 & 0.145 \\
\hline $01 t$ & 0.320 & -1.857 & 15.484 & 1.181 & -5.16 & -2.16 & -0.266 & -1.681 & 0.166 & 0.381 & 2017 & & 205.2 & & & 283.7 & 0.495 & 0.202 \\
\hline $02 \mathrm{c}$ & 0.383 & -1.837 & 15.746 & 1.224 & -5.21 & -2.28 & -0.252 & -1.633 & 0.029 & 0.313 & 2009 & 1888 & 203.8 & 207.5 & 212.3 & 289.0 & 0.532 & 0.105 \\
\hline $02 t$ & 0.281 & -1.981 & 15.386 & 1.001 & -5.07 & -1.98 & -0.269 & -1.681 & 0.100 & 0.356 & 2019 & & 205.0 & & & 278.5 & 0.543 & 0.169 \\
\hline $03 \mathrm{c}$ & 0.268 & -2.238 & 15.354 & 0.925 & -5.05 & -1.85 & -0.204 & -1.613 & 0.102 & 0.189 & 2002 & 1870 & 204.6 & 208.2 & 210.6 & 245.2 & 0.428 & -0.004 \\
\hline $04 c$ & 0.245 & -2.317 & 15.314 & 0.849 & -4.99 & -1.91 & -0.222 & -1.609 & 0.044 & 0.161 & 2001 & 1875 & 204.9 & 208.4 & 210.1 & 228.8 & 0.443 & -0.034 \\
\hline 05 & -0.033 & -2.110 & 13.968 & 0.996 & -4.715 & -1.831 & -0.285 & -1.685 & 0.145 & 0.338 & 1966 & & 208.2 & 217.0 & & 284.4 & 0.444 & 0.192 \\
\hline 06 & -0.049 & -2.223 & 13.890 & 0.791 & -4.679 & -1.656 & -0.310 & -1.697 & 0.071 & 0.322 & 1962 & & 208.1 & 217.8 & & 279.1 & 0.458 & 0.152 \\
\hline 07 & -0.116 & -2.444 & 13.316 & 0.782 & -4.489 & -1.479 & -0.206 & -1.698 & 0.134 & 0.183 & 1916 & & 208.7 & 216.9 & & & 0.357 & 0.055 \\
\hline 08 & -0.152 & -2.530 & 13.662 & 0.585 & -4.400 & -1.361 & -0.172 & -1.696 & 0.068 & 0.177 & 1914 & & 202.9 & 218.5 & & & 0.451 & 0.008 \\
\hline 09c & 0.419 & -1.827 & 16.484 & 1.549 & -5.584 & -2.592 & -0.413 & -1.808 & 0.140 & 0.380 & $1996^{b}$ & 1889 & 203.3 & 206.2 & 209.8 & 295.8 & 0.490 & 0.169 \\
\hline $10 \mathrm{c}$ & 0.384 & -1.954 & 16.075 & - & -5.478 & -2.468 & -0.418 & -1.814 & 0.078 & 0.358 & $2000^{b}$ & 1893 & 203.6 & 207.2 & 210.0 & 288.0 & 0.543 & 0.136 \\
\hline $11 \mathrm{c}$ & 0.229 & -2.289 & 15.292 & 1.188 & -5.379 & -2.089 & -0.373 & -1.792 & 0.131 & 0.208 & $1993^{b}$ & 1902 & 201.8 & 204.7 & 209.3 & 247.5 & 0.404 & 0.024 \\
\hline $11 t$ & 0.384 & -2.154 & 15.295 & 1.184 & -5.284 & -2.038 & -0.359 & -1.843 & 0.120 & 0.223 & $2024^{b}$ & & 206.2 & & & 249.1 & 0.409 & 0.043 \\
\hline $12 c$ & 0.248 & -2.398 & 15.567 & 0.979 & -5.348 & -2.043 & -0.389 & -1.800 & 0.056 & 0.178 & $1984^{b}$ & 1876 & 203.6 & 204.2 & 208.0 & 229.2 & 0.464 & -0.020 \\
\hline $12 t$ & 0.408 & -2.250 & 15.533 & 1.003 & -5.287 & -2.042 & -0.400 & -1.860 & 0.052 & 0.191 & $2020^{b}$ & & 205.2 & & & 223.3 & 0.463 & -0.002 \\
\hline $\mathbf{A}$ & 0.728 & -1.564 & 18.538 & 1.457 & -5.907 & -2.873 & -0.206 & -1.710 & 0.140 & 0.382 & 2066 & 1916 & 197.6 & & 202.5 & 290.7 & 0.489 & 0.168 \\
\hline B & 0.697 & -1.645 & 17.972 & 1.441 & -5.798 & -2.725 & -0.216 & -1.716 & 0.072 & 0.359 & 2067 & 1920 & 197.4 & & 203.4 & 284.8 & 0.542 & 0.132 \\
\hline C & 0.562 & -2.098 & 17.088 & 1.224 & -5.642 & -2.311 & -0.179 & -1.684 & 0.127 & 0.209 & 2061 & 1906 & 198.7 & & 203.5 & $245.7,235.0$ & 0.401 & 0.029 \\
\hline D & 0.620 & -2.200 & 17.974 & 0.995 & -5.621 & -2.288 & -0.209 & -1.695 & 0.052 & 0.178 & 2059 & 1908 & 198.4 & & 202.4 & $225.7,219.3$ & 0.432 & -0.014 \\
\hline $\mathbf{F}$ & 0.422 & -2.074 & 16.921 & 1.106 & -5.558 & -2.223 & -0.202 & -1.690 & 0.127 & 0.207 & 2059 & 1916 & 198.5 & & 202.7 & 237.6 & 0.410 & 0.027 \\
\hline G & 0.470 & -2.178 & 17.950 & 0.897 & -5.544 & -2.169 & -0.224 & -1.698 & 0.049 & 0.175 & 2058 & 1910 & 198.6 & & 202.3 & 223.2 & 0.459 & -0.016 \\
\hline H & 0.005 & -2.203 & 16.296 & 1.053 & -4.824 & -2.184 & -0.078 & -1.291 & 0.105 & 0.171 & 2008 & 1906 & 205.8 & 213.3 & 216.0 & 246.9 & 0.418 & -0.009 \\
\hline I & 0.020 & -2.330 & 16.571 & 0.791 & -4.831 & -2.093 & -0.095 & -1.291 & 0.022 & 0.143 & 2006 & 1904 & 206.0 & 214.0 & 215.8 & 235.4 & 0.468 & -0.058 \\
\hline
\end{tabular}

${ }^{a}$ Collected as KBr pellets unless otherwise specified; ${ }^{b}$ Collected in hexane 


\subsubsection{Relationships}

Further support that the first oxidation process of 1-12 is related to the HOMO that is mainly metal-based, the relationship between the first oxidation potential (in V) and the DFT calculated HOMO energy, $E_{\mathrm{HOMO}}$ in $\mathrm{eV}$, is illustrated in Figure 11. Both $E_{\mathrm{HOMO}}$ and the DFT calculated two-electron ionization potential, $I P_{2 \mathrm{e}-}$, relate linearly to the first oxidation process:

$$
\begin{array}{ll}
E_{\mathrm{pa}}=-0.53 E_{\mathrm{HOMO}}-2.44 & \left(\mathrm{R}^{2}=0.84\right) \\
E_{\mathrm{pa}}=0.21 I P_{2 \mathrm{e}-}-2.94 & \left(\mathrm{R}^{2}=0.91\right)
\end{array}
$$

$I P_{2 \mathrm{e}-}$ is obtained from the difference in the DFT calculated electronic energies of the neutral and the doubly oxidized complexes $\mathbf{1 - 8}$, the energy needed to oxidize $\mathrm{W}(0)$ to W(II).
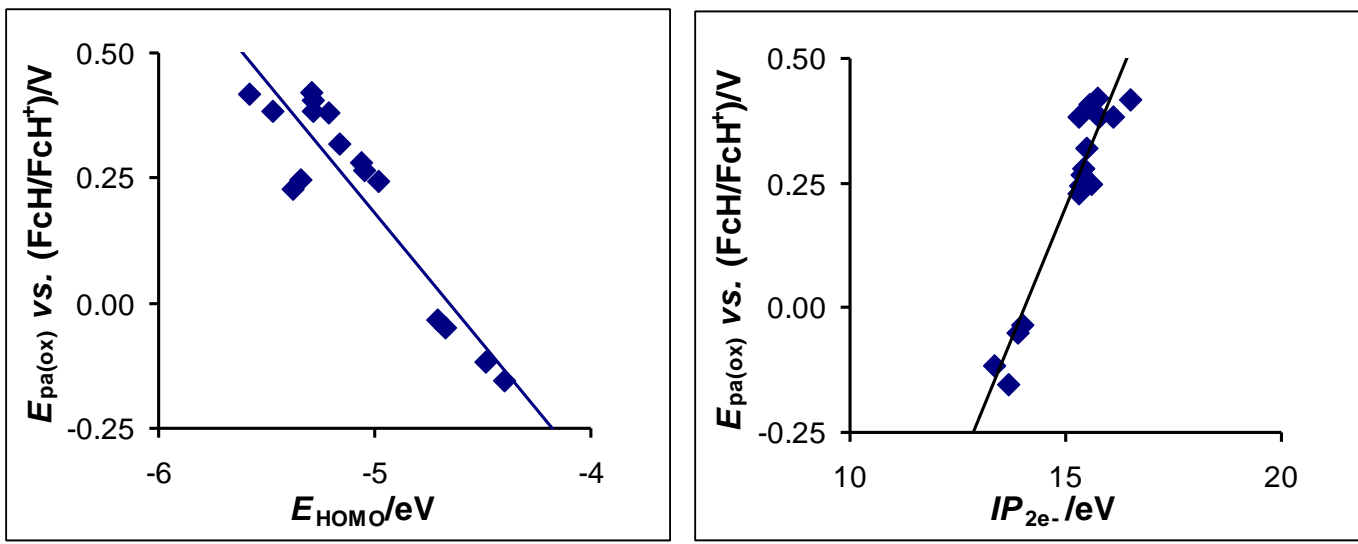

Figure 11. Linear relationship between the DFT calculated (a) $E_{\mathrm{HOMO}}$ and the experimental oxidation potential $E_{\mathrm{pa}}$ and (b) the two-electron ionization potential, $I P_{2 \mathrm{e}}$, and the experimental oxidation potential $E_{\mathrm{pa}}$ of complexes 1-12. Data are in Table 1 and Table 2. Experimental potentials are reported relative to the $\mathrm{FcH} / \mathrm{FcH}^{+}$couple.

The trend obtained between the DFT calculated LUMO energy, $E_{\mathrm{LUMO}}$ in $\mathrm{eV}$, of neutral 1-12, A-D and F-I and the reduction potential $E_{\mathrm{pc}}$, confirms the role of the LUMO, that is mainly centred on the carbene ligand, in the reduction process. An electron will more easily be added to a LUMO of lower, more negative energy with a higher electron affinity $E A$ (in $\mathrm{eV}$ ), i.e. the complex will more easily be reduced at a higher, less negative reduction potential, see Figure 12. 

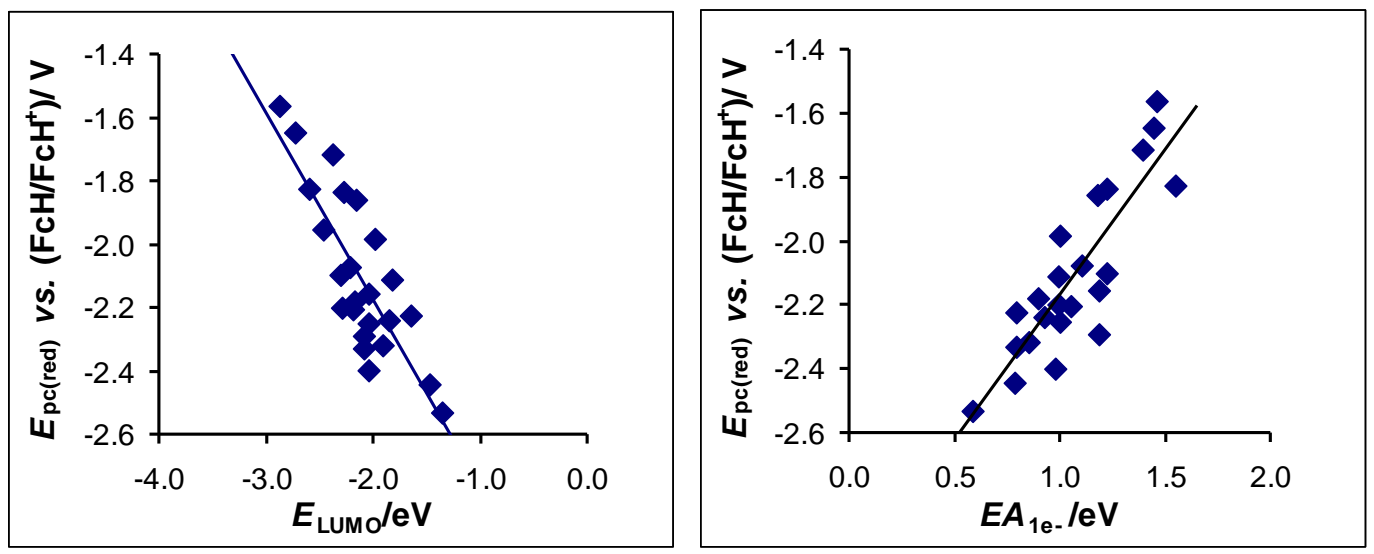

Figure 12. Trend between the DFT calculated (a) $E_{\mathrm{LUMO}}$ and the experimental reduction potential $E_{\mathrm{pc}}$ and (b) electron affinity, $E A$, and the experimental reduction potential $E_{\mathrm{pc}}$ of complexes 1-12, A-D and F-I. Data are in Table 2.

The effect of $\mathrm{CO}$ substitution in pentacarbonyl Fischer W-carbene complexes on the ease of oxidation, as related to the HOMO energy, $E_{\mathrm{HOMO}}$ in $\mathrm{eV}$, over a large potential range $\left(-0.2-0.8 \mathrm{~V}\right.$ vs. $\left.\mathrm{FcH} / \mathrm{FcH}^{+}\right)$is illustrated in Figure 13 for the sixteen $\mathrm{W}$-carbene complexes in Table 1 and given by:

$$
E_{\mathrm{pa}}\left(\mathrm{V} v s . \mathrm{FcH} / \mathrm{FcH}^{+}\right)=-0.58 E_{\mathrm{HOMO}}(\mathrm{eV})-2.73 \quad\left(\mathrm{R}^{2}=0.91\right)
$$

It is clear that the systematic substitution of a CO group in pentacarbonyl Fischer Wcarbene complexes, with a ligand of less $\pi$-acceptor ability, results in a more stable $\mathrm{HOMO}$ of mainly $\mathrm{d}_{\pi}$-character (Figure 8).

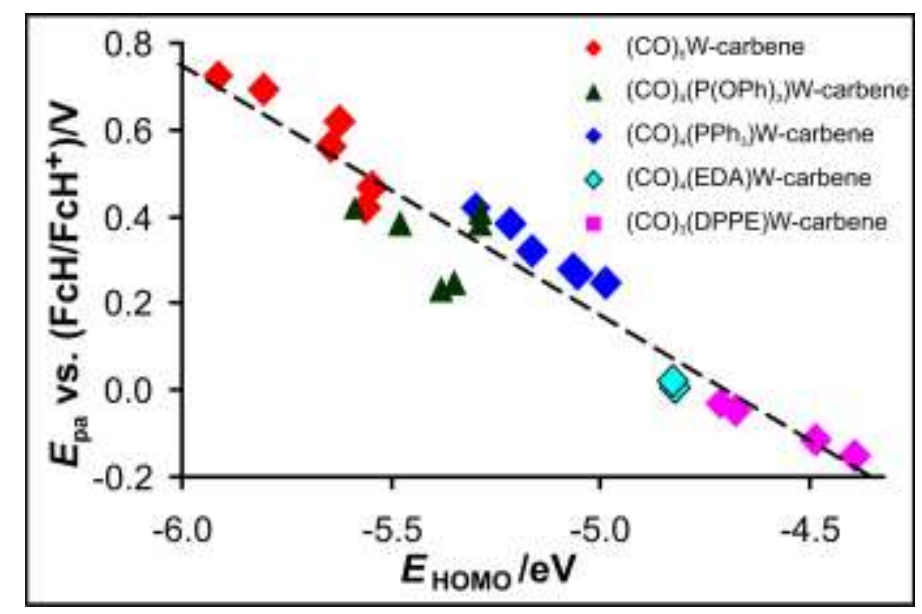

Figure 13. Linear relationship between the DFT calculated $E_{\mathrm{HOMO}}$ and the experimental oxidation potential $E_{\mathrm{pa}}$ of complexes 1-12 and A-D, F-I. Data are in Table 1, Table 2 and from reference [4]. Experimental potentials are reported relative to the $\mathrm{FcH} / \mathrm{FcH}^{+}$couple. 


\section{Conclusions}

Novel phosphine-substituted carbene complexes of tungsten(0) were synthesized by substituting one or two carbonyl ligands in pentacarbonyl Fischer W-carbene complexes. Both cis- and trans-isomers of the monosubstituted complexes were obtained, while mer-substituted complexes were isolated for the disubstituted complexes. The proposed conformations were confirmed with X-ray crystallography. Electrochemical studies provided an experimental way to measure the relative reactivity of the complexes towards oxidation and reduction. DFT calculations showed that the oxidation is $\mathrm{W}$-metal based and that the reduction is centred on the carbene ligand. Ligands attached directly to the W-metal, influence the $\mathrm{W}(0)$ to $\mathrm{W}(\mathrm{II})$ oxidation process to a larger extent than different carbene ligand substituents. The oxidation potential of the first $\mathrm{W}$-metal centred oxidation process of 1-12 covers a range of $0.57 \mathrm{~V}$, from -0.15 to $0.42 \mathrm{~V}$, while the reduction potential of the reduction process of the carbene carbon is found over a large range of $0.78 \mathrm{~V}$, from -2.53 to $1.75 \mathrm{~V}$. The influence of the nitrogen or oxygen heteroatom and the furyl or thienyl substituents on the charge of the carbene carbon is reflected by the large range of reduction potentials of the reduction process $(\sim 0.780 \mathrm{~V})$ measured for the phosphineand phosphite-substituted tungsten(0) Fischer carbene complexes of this study.

\section{Acknowledgements}

This work has received support from the Norwegian Supercomputing Program (NOTUR) through a grant of computer time (Grant No. NN4654K) (JC), the South African National Research Foundation (JC) and the Central Research Fund of the University of the Free State, Bloemfontein (JC) and the University of Pretoria (ML and PHvR).

\section{Supporting Information}

Cif files containing the crystallographic data for this paper, optimized coordinates of the DFT calculations and selected structural data are available with the on-line version only. 


\section{References}

[1] M. Landman, R. Liu, P.H. van Rooyen, J. Conradie, Electrochemistry of Fischer alkoxycarbene complexes of chromium: The use of DFT to predict and understand oxidation and reduction potentials, Electrochimica Acta 114 (2013), 205-214.

[2] B. van der Westhuizen, P.J. Swarts, L.M. van Jaarsveld, D.C. Liles, U. Siegert, J.C. Swarts, I. Fernández, D.I. Bezuidenhout, Substituent Effects on the Electrochemical, Spectroscopic, and Structural Properties of Fischer Mono- and Biscarbene Complexes of Chromium(0), Inorg. Chem. 52 (2013) 6674-6684.

[3] R. Metelková, T. Tobrman, H. Kvapilová, I. Hoskovcová, J. Ludvík, Synthesis, characterization and electrochemical investigation of hetarylchromium(0) aminocarbene complexes, Electrochim. Acta 82 (2012) 470-477.

[4] M Landman, R. Pretorius, B.E. Buitendach, P.H. van Rooyen, J. Conradie, Synthesis, structure and electrochemistry of Fischer alkoxy- and aminocarbene complexes of tungsten: The use of DFT to predict and understand oxidation and reduction potentials, Organometallics 32 (2013) 5491-5503.

[5] O.S. Mills, A.D. Redhouse, The structure of methylmethoxycarbene-triphenylphosphinetetracarbonylchromium, Chemical Communications (London) 22 (1966) 814-815.

[6] H. Werner, H. Rascher, The synthesis of tetracarbonyl(tertiary phosphine) methoxycarbenechromium complexes, Inorganica Chimica Acta 2 (1968) 181-185.

[7] E.O. Fischer, R. Aumann, Übergangsmetall-Carben-Komplexe, XI. Über die Darstellung von cis$\mathrm{M}\left(\mathrm{C}_{6} \mathrm{H}_{5}\right)_{3}$-Methoxy-organylcarben-metall-tetracarbonyl-Komplexen $(\mathrm{M}=\mathrm{P}, \mathrm{As}, \mathrm{Sb})$ des Chroms, Molybdäns und Wolframs aus den $\mathrm{M}\left(\mathrm{C}_{6} \mathrm{H}_{5}\right)_{3}$-Metall-pentacarbonylen durch $\mathrm{CH}_{3}{ }^{-} / \mathrm{CH}_{3}{ }^{+}$-Addition, Chemische Berichte 102 (1969) 1495-1503.

[8] C. Crotti, E. Farnetti, T. Celestino, M. Stener, S. Fontana, Donor Properties of Diphosphine Ligands in Tungsten Carbonyl Complexes: Synchrotron Radiation XPS Measurements and DFT Calculations, Organometallics 23 (2004) 5219-5225.

[9] T.L. Brown, K.J. Lee, Ligand steric properties, Coordination Chemistry Review 128 (1993) 89-116.

[10] W.A. Herrmann, L.J. Goossen, G.R.J. Artus, C. Köcher, Metal Complexes of Chiral Imidazolin-2ylidene Ligands, Organometallics 16 (1997) 2472-2477.

[11] I. Fernández, M.A. Sierra, M. Gómez-Gallego, M.J. Mancheño, F.P. Cossío, The Photochemical Reactivity of the "Photo-Inert" Tungsten (Fischer) Carbene Complexes, Angewandte Chemie 118 (2005) 131-134.

[12] S. Maiorana, P. Seneci, T. Rossi, C. Baldoli, M. Ciraco, E. de Magistris, E. Licandro, A. Papagni, S. Provera, Synthesis of polymer-bound Fischer chromium alkoxy and aminocarbene complexes, Tetrahedron Letters 40 (1999) 3635-3638.

[13] S. Klapdohr, K.H. Dötz, W. Assenmacher, W. Hoffbauer, N. Hüsing, M. Nieger, J. Pfeiffer, M. Popall, U. Schubert, G. Trimmel, Incorporation of Chromium Carbenes in a Silica Matrix by SolGel Processing: Application to Aminolysis of Alkoxycarbene Complexes, Chemical European Journal 6 (2000) 3006-3017. 
[14] M.D. Cooke, E.O. Fischer, Transition metal carbene complexes : LIII. Proof of the absence of a free carbene in the preparation of a cyclopropane from a metal-carbene complex, Journal for Organometallic Chemistry 56 (1973) 279-284.

[15] J. Barluenga, K. Muniz, M. Tomas, A. Ballesteros, S. Garcia-Granda, A Simple Approach toward Enantiomerically Pure Fischer Carbene Complexes of Chromium and Molybdenum: Chiral Modification of the Metal Fragment, Organometallics 22 (2003) 1756-1760.

[16] A. Arrieta, F.P. Cossío, I. Fernández, M. Gomez-Gallego, B. Lecea, M.J. Mancheno, M.A. Sierra, A Theoretical-Experimental Approach to the Mechanism of the Photocarbonylation of Chromium(0) (Fischer)-Carbene Complexes and Their Reaction with Imines, Journal of the American Chemical Society 122 (2000) 11509-11510.

[17] E.W. Reinheimer, K.A. Kantardjieff, S.R. Herron, C.G. Tisserat, J.A. Casalnuovo, Crystal structures of diphosphinated group 6 Fischer alkoxy carbenes, Journal of Chemical Crystallography 33 (2003) 503-514.

[18] G.A. Mabbott, An introduction to cyclic voltammetry, Journal of Chemical Education 60 (1983) 697-702.

[19] P.T. Kissinger, W.R. Heineman, Cyclic voltammetry, Journal of Chemical Education 60 (1983) 702-706.

[20] J.J. van Benschoten, J.Y. Lewis, W.R. Heineman, D.A. Roston, P.T. Kissinger, Cyclic voltammetry experiment, Journal of Chemical Education 60 (1983) 772-776.

[21] J.J.C. Erasmus, J. Conradie, Chemical and electrochemical oxidation of $[\mathrm{Rh}(\beta-$ diketonato $\left.)(\mathrm{CO})\left(\mathrm{P}\left(\mathrm{OCH}_{2}\right)_{3} \mathrm{CCH}_{3}\right)\right]$ : an experimental and DFT study, Dalton Transactions 42 (2013) 8655-8666.

[22] J. Conradie, Oxidation potential of $\left[\mathrm{Rh}(\beta\right.$-diketonato $\left.)\left(\mathrm{P}(\mathrm{OPh})_{3}\right)_{2}\right]$ complexes - relationships with experimental, electronic and calculated parameters, Electrochimica Acta 110 (2013) 718-725.

[23] H. Ferreira, M.M. Conradie, J. Conradie, Electrochemical study of carbonyl phosphine $\beta$ diketonatorhodium(I) complexes, Electrochimica Acta 13 (2013) 519- 526.

[24] D.F. Schriver, M.A. Drezdzon, The manipulation of Air-Sensitive Compounds, $2^{\text {nd }}$ ed., Wiley, New York, 1980.

[25] G.H. Spies, R.J. Angelici, Model studies of thiophene hydrodesulfurization using (.eta.thiophene)Ru(.eta.- $\left.\mathrm{C}_{5} \mathrm{H}_{5}\right)^{+}$. Reactions leading to $\mathrm{C}-\mathrm{S}$ bond cleavage, Organometallics 6 (1987) 1897-1903.

[26] H. Meerwein, Triethyloxonium fluoroborate [Oxonium compounds, triethyloxonium tetrafluoroborate], Organic Syntheses 46 (1966) 113-113.

[27] S. Aoki, T. Fujimura, E. Nakamura, A protective strategy in carbene complex chemistry. Synthesis of functionalized Fischer carbene complexes via dianion formation, Journal of the American Chemical Society 114 (1992) 2985-2990.

[28] C. Crause, H. Görls, S. Lotz, Binuclear biscarbene complexes of furan, Dalton Transactions 9 (2005) 1649-1657. 
[29] D.T. Sawyer, J.L. Roberts Jr., Experimental Electrochemistry for Chemists, Wiley, New York, 1974, p.54.

[30] G. Gritzner, J. Kuta, Recommendations on reporting electrode potentials in nonaqueous solvents, Pure and Applied Chemistry 56 (1984) 461-466.

[31] A.J.L. Pombeiro, Electron-donor/acceptor properties of carbynes, carbenes, vinylidenes, allenylidenes and alkynyls as measured by electrochemical ligand parameters, Journal of Organometallic Chemistry 690 (2005) 6021-6040.

[32] A.D. Becke, Density-functional exchange-energy approximation with correct asymptotic behavior, Physical Review A 38 (1988) 3098-3100.

[33] C.T. Lee, W.T. Yang, R.G. Parr, Development of the Colle-Salvetti correlation-energy formula into a functional of the electron-density, Physical Review B 37 (1988) 785-789.

[34] M.J. Frisch, G.W. Trucks, H.B. Schlegel, G.E. Scuseria, M.A. Robb, J.R. Cheeseman, G. Scalmani, V. Barone, B. Mennucci, G.A. Petersson, H. Nakatsuji, M. Caricato, X. Li, H.P. Hratchian, A.F. Izmaylov, J. Bloino, G. Zheng, J.L. Sonnenberg, M. Hada, M. Ehara, K. Toyota, R. Fukuda, J. Hasegawa, M. Ishida, T. Nakajima, Y. Honda, O. Kitao, H. Nakai, T. Vreven, J.A. Montgomery (Jr), J.E. Peralta, F. Ogliaro, M. Bearpark, J.J. Heyd, E. Brothers, K.N. Kudin, V.N. Staroverov, T. Keith, R. Kobayashi, J. Normand, K. Raghavachari, A. Rendell, J.C. Burant, S.S. Iyengar, J. Tomasi, M. Cossi, N. Rega, J.M. Millam, M. Klene, J.E. Knox, J.B. Cross, V. Bakken, C. Adamo, J. Jaramillo, R. Gomperts, R.E. Stratmann, O. Yazyev, A.J. Austin, R. Cammi, C. Pomelli, J.W. Ochterski, R.L. Martin, K. Morokuma, V.G. Zakrzewski, G.A. Voth, P. Salvador, J.J. Dannenberg, S. Dapprich, A.D. Daniels, O. Farkas, J.B. Foresman, J.V. Ortiz, J. Cioslowski, D.J. Fox, Gaussian 09, Revision C.01, Gaussian Inc., Wallingford CT, 2010.

[35] F. Weigend, R. Ahlrichs, Balanced basis sets of split valence, triple zeta valence and quadruple zeta valence quality for $\mathrm{H}$ to Rn: Design and assessment of accuracy, Physical Chemistry Chemical Physics 7 (2005) 3297-3305.

[36] NBO 3.1. E.D. Glendening, J.K. Badenhoop, A.E. Reed, J.E. Carpenter, J.A. Bohmann, C.M. Morales, F. Weinhold (Theoretical Chemistry Institute, University of Wisconsin, Madison, WI, 2001).

[37] APEX2 (including SAINT and SADABS), Bruker AXS Inc., Madison, WI, 2012.

[38] G.M. Sheldrick, A short history of SHELX, Acta Crystallographica A 64 (2008) 112-122.

[39] L.J. Farrugia, ORTEP-3 for Windows - a version of ORTEP-III with a Graphical User Interface (GUI), Journal of Applied Crystallography 30 (1997) 565-565.

[40] E.O. Fischer, H. Fischer, Übergangsmetall-Carben-Komplexe, LXVIII. Synthese von cis- und trans-Tetracarbonyl(carben)(phosphin)-Komplexen von $\operatorname{Chrom}(0)$ und Wolfram(0), Chemische Berichte 107 (1974) 657-672.

[41] R. Streubel, S. Priemer, J. Jeske, P.G. Jones, Stereochemically defined metal carbene complexes as chemical probes for studies of the ring formation of $2 \mathrm{H}$-azaphosphirene complexes, Journal of Organometallic Chemistry 618 (2001) 423-434. 
[42] M. Landman, J. Ramontja, M. van Staden, D.I. Bezuidenhout, P.H. van Rooyen, D.C. Liles, S. Lotz, Properties of homo- and heteronuclear mixed biscarbene complexes with conjugated bithiophene units, Inorganica Chimica Acta 363 (2010) 705-717.

[43] M. Landman, H. Görls and S. Lotz, Chromium and Tungsten Carbene Complexes of Thieno[3,2b]thiophene, European Journal of Inorganic Chemistry (2001) 233-238.

[44] S. Lotz, M. Landman, H. Görls, C. Crause, H. Nienaber, A. Olivier, Di-tungsten Bis-carbene Complexes Linked by Condensed Heteroaromatic Spacers, Zeitschrift für Naturforschung 62b (2007) 419-426.

[45] E.O. Fischer, H. Fischer, H. Werner, Demonstration of Isomers and Intramolecular Isomerization of Carbene Complexes of Chromium, Angewandte Chemie International Edition (English) 11 (1972) 644-645.

[46] M.A. Sierra, I. Fernández, M. Gomez-Gallego, M.R. Torres, F.P. Cossi, A. Arrieta, B. Lecea, A. Poveda, J. Jimenez-Barbero, Light-Induced Aminocarbene to Imine Dyotropic Rearrangement in a Chromium(0) Center: An Unprecedented Reaction Pathway, Journal of the American Chemical Society 125 (2003) 9572-9573.

[47] E.O. Fischer, H. Fischer, F.R. Kreissl, Übergangsmetall-Carben-Komplexe : LXX. Über die Abreaktion von Pentacarbonyl-phosphin(methoxymethylmethylen)-Metall-Komplexen, Journal of Organometallic Chemistry 64 (1974) C41-44.

[48] G.T. Andrews, I.J. Colquhoun, W. McFarlane, Fourier transform heteronuclear magnetic triple resonance in complex spin systems-III: Symmetrical ditertiary phosphine complexes of group VI metal carbonyls, Polyhedron 2 (1983) 783-790.

[49] J.L. Bookham, W. McFarlane, M. Thornton-Pett, S. Jones, Stereoselective addition reactions of diphenylphosphine: meso- and rac-1,2-diphenyl-1,2-bis(diphenylphosphino)ethane and their Group 6 metal tetracarbonyl complexes. Crystal structures of the molybdenum derivatives, Journal of the Chemical Society, Dalton Transactions 12 (1990) 3621-3627.

[50] G.T. Andrews, I.J. Colquhoun, W. McFarlane, S.O. Grim, Transition-metal chemical shifts in complexes of molybdenum(0) and tungsten(0), Journal of the Chemical Society, Dalton Transactions 12 (1982) 2353-2358.

[51] J.A. Connor, E.M. Jones, E.W. Randall, E. Rosenberg, The ${ }^{13}$ C nuclear magnetic resonance spectra of carbene and isonitrile complexes of chromium and tungsten and a reinvestigation of the ${ }^{1} \mathrm{H}$ nuclear magnetic resonance spectra of phenyl carbene complexes, Journal of the Chemical Society, Dalton Transactions 22 (1972) 2419-2424.

[52] J.A. Connor, E.M. Jones, Stabilisation of nucleophilic carbenes co-ordinated to transition metals, Journal of the Chemical Society (A) (1971) 1974-1979.

[53] P.S. Braterman, D.W. Milne, E.W. Randall, E. Rosenberg, Carbon-13 nuclear magnetic resonance spectra of tungsten and molybdenum carbonyl derivatives, Journal of the Chemical Society, Dalton Transactions 10 (1973) 1027-1031. 
[54] M.F. Guns, E.G. Claeys, G.P. van der Kelen, Vibrational study in the low frequency region (700$100 \mathrm{~cm}-1)$ of phosphine monosubstituted group VI B metal carbonyls, Journal of Molecular Structure 65 (1980) 3-17.

[55] W. Buchner, W.A. Schenk, Carbon-13 NMR spectra of monosubstituted tungsten carbonyl complexes. NMR trans influence in octahedral tungsten (0) compounds, Inorganic Chemistry 23 (1984) 132-137.

[56] M.Y. Darensbourg, D.J. Darensbourg, Spectroscopic studies of some carbene pentacarbonyl complexes of chromium(0) and tungsten(0), Inorganic Chemistry 9 (1970) 32-39.

[57] U. Schubert, Structural consequences of bonding in transition metal carbene complexes, Coordination Chemistry Reviews 55 (1984) 261-286.

[58] A. Hafner, L.S. Hegedus, G. de Week, B. Hawkins, K.H. Dötz, Chromium-53 nuclear magnetic resonance studies of pentacarbonylchromium carbene complexes, Journal of the American Chemical Society 110 (1988) 8413-8421.

[59] D.J. Cardin, B. Cetinkaya, M.F. Lappert, Transition metal-carbene complexes, Chemical Reviews 72 (1972) 545-574.

[60] C. Baldoli, P. Cerea, L. Falciola, C. Giannini, E. Licandro, S. Maiorana, P. Mussini, D. Perdicchia, The electrochemical activity of heteroatom-stabilized Fischer-type carbene complexes, Journal of Organometallic Chemistry 690 (2005) 5777-5787.

[61] I. Hoskovcová, J. Roháčová, D. Dvořák, T.Tobrman, S.Záliš, R. Zvěřinová, J. Ludvík, Synthesis and electrochemical study of iron, chromium and tungsten aminocarbenes: Role of ligand structure and central metal nature, Electrochimica Acta 55 (2010) 8341-8351.

[62] M.J. Cook, I. Chambrier, G.F. White, E. Fourie, J.C. Swarts, Electrochemical and EPR studies of two substituted bis-cadmium tris-phthalocyanine complexes: elucidation of unexpectedly different free-radical character, Dalton Transactions 7 (2009) 1136-1144.

[63] E. Fourie, J.C. Swarts, I. Chambrier, M.J Cook, Electrochemical and spectroscopic detection of self-association of octa-alkyl phthalocyaninato cadmium compounds into dimeric species, Dalton Transactions 7 (2009) 1145-1154.

[64] K.F. Purcell, J.C. Kotz, Inorganic Chemistry, W.B. Saunders Company, Philadelphia, 1977, p.59.

[65] A.M. Bond, R. Colton, J.E. Kevekordes, Redox Reactions of Chromium Tetracarbonyl and Tricarbonyl Complexes: Thermodynamic, Kinetic, and Catalytic Aspects of Isomerization in the fac / mer -Tricarbonyltris(trimethyl phosphite)chromium(1+/0) System, Inorganic Chemistry 25 (1986) 749-756. 